\title{
Towards Language-Based Mitigation of Traffic Analysis Attacks
}

\author{
Jeppe Fredsgaard Blaabjerg \\ Aarhus University \\ jfblaa@cs.au.dk
}

\author{
Aslan Askarov \\ Aarhus University \\ aslan@cs.au.dk
}

\begin{abstract}
Traffic analysis attacks pose a major risk for online security. Distinctive patterns in communication act as fingerprints, enabling adversaries to de-anonymise communicating parties or to infer sensitive information. Despite the attacks being known for decades, practical solution are scarce. Network layer countermeasures have relied on black box padding schemes that require significant overheads in latency and bandwidth to mitigate the attacks, without fundamentally preventing them, and the problem has received little attention in the language-based information flow literature. Language-based methods provide a strong foundation for fundamentally addressing security issues, but previous work has overwhelmingly assumed that interactive programs communicate over secure channels, where messages are undetectable by unprivileged adversaries. This assumption is too strong for online communication where packets can be trivially observed by eavesdropping. In this paper we introduce SELENE, a small language for principled, provably secure communication over channels where packets are publicly observable, and we demonstrate how our program level defence can reduce the latency and bandwidth overheads induced compared with program-agnostic defence mechanisms. We believe that our results constitute a step towards practical, secure online communication.
\end{abstract}

Index Terms-Traffic analysis, noninterference, languagebased security

\section{INTRODUCTION}

Work on traffic analysis attacks has shown that many systems and services are vulnerable to de-anonymisation and loss of secrecy by producing distinctive patterns in their network traffic. Traffic analysis has particularly been studied in the context of anonymous communication and website fingerprinting [12, 16, 22, 23, 28, 29, 32]. Defence strategies against website fingerprinting are commonly done at the network level [12], and rely on constant rate padding, where source traffic is morphed to fit a predefined target pattern [19]. Constant rate padding can be applied in a black box fashion, making it an intuitively appealing technique against website fingerprinting. However, it often falls short in practice as achieving a high degree of security introduces intolerable bandwidth and latency overheads for many applications [17], such as anonymous, low-latency browsing and communication [18] and privacy preserving IoT devices [1]. Cherubin et al. argue that the application layer defences against traffic analysis are more natural as they act directly on the objects that are fingerprinted at the network level, while defences at lower layers must model legitimate traffic in order to generate convincing traffic padding [12].
Website fingerprinting is not the only attack made possible by traffic analysis. Online services that process sensitive information are now ubiquitous and previous work has shown that many such services are vulnerable to attack, as their communication behaviour reveals system secrets. Analysis by Chen et al. suggests that the scope of the issue is industrywide [11]. Their study finds that design features used for creating reactive sites generate characteristic traffic patterns that allow an adversary to infer highly detailed, sensitive user information. They demonstrate this vulnerability across a number of high-profile websites, e.g. they are able to infer which illness a user selects on an online health site, and argue that traffic analysis attacks pose an unprecedented threat to the confidentiality of user information processed by online systems, and that this information is often far more sensitive than identifying which website a user visits as studied in anonymity research.

Language-based information flow methods provide principled ways of enforcing that the observable behaviour of a program does not depend on secrets. The language-based approach is appealing as the security condition of noninterference [20] can be provably enforced using a type system. O'Neill et al. formulate a noninterference condition for interactive programs [27], where programs communicate over inand output channels. Their condition requires that input on secret channels does not influence output on public channels. This condition has been used in a breadth of other work $[3,8,10,13,14,21,31]$. Unfortunately, the models used in these works assume that messages on secret channels are invisible to adversaries. Other work models Internet communication using expressly public channels [25]. This makes the security results inapplicable for reasoning about online services and distributed programs where secret information is shared between remote, trusted entities. The only other work we are aware of, that allows an adversary to observe the communication behaviour of a program on non-public channels, is by Sabelfeld and Mantel [30]. They consider encrypted channels that protect message contents, but do not hide message presence, and give a timing-sensitive security condition. Their security condition does not consider the size of messages, which can be exploited in traffic analysis attacks, and their semantics lets the blocking behaviour of receives on encrypted channels be public by letting number of available messages be public. 
for the patient to send the result to the doctor. In this case, or in case the test result is negative, nothing will be queued to the clinic before the send occurs. If the queue is empty at the time of a scheduled send, dummy packets are sent instead. When, to whom, and how much the doctor sends is thereby made public, while what the doctor sends is kept secret.

As a second example, we consider the password checker in Program 6.

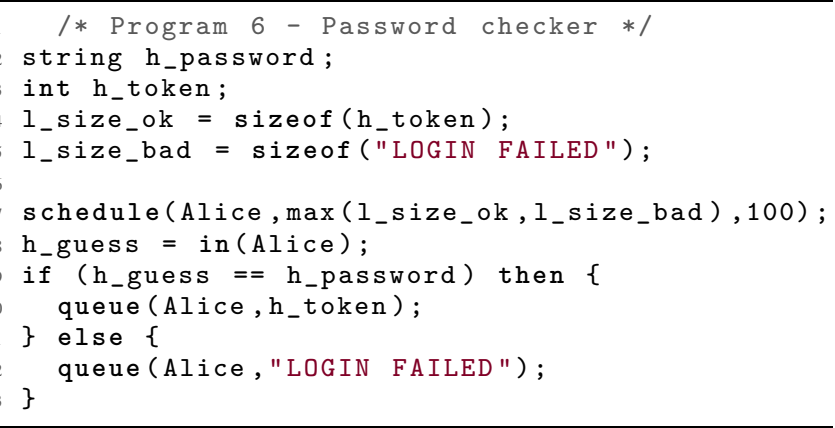

The password checker stores a secret password and returns a token to be used as proof of authority upon receiving a successful guess. The password checker schedules bandwidth for sending either the token or a login failure message by using the maximum of the two sizes. By scheduling the response before the guess is received, the program does not leak whether a valid guess was received, let alone whether the guess was correct.

As a final example, we consider a small popularity poll. Alice wishes to know whether her opinion that dogs are better than cats is shared by a majority of people. She sets up a simple online voting service running Program 7 below:

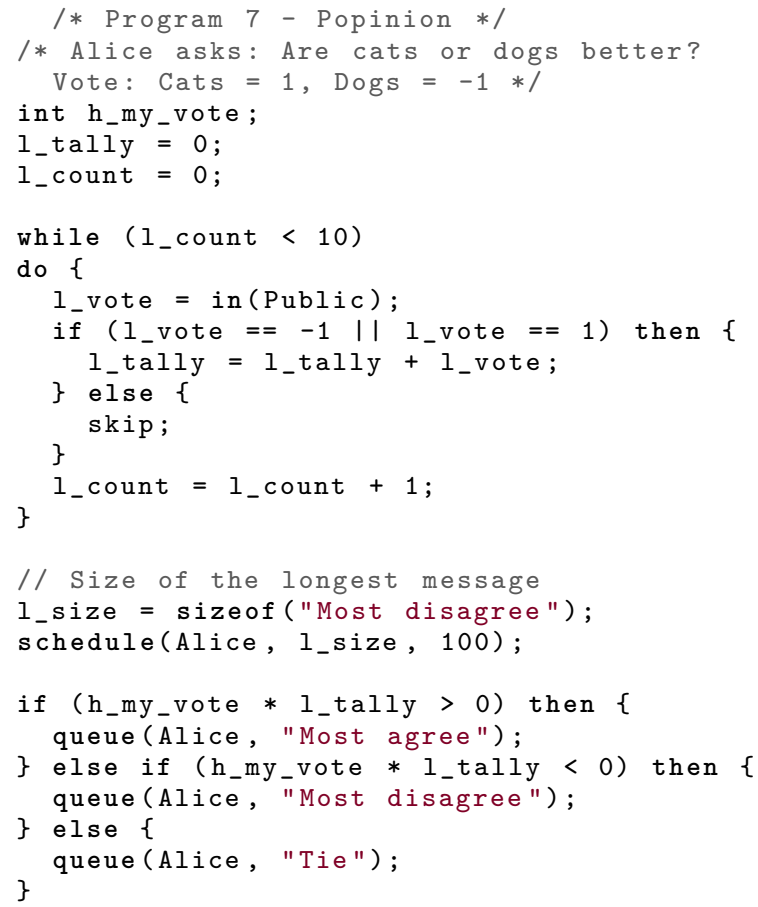

The voting service stores Alice's secret choice in variable h_my_vote, tallies ten votes from a public channel, and schedules sending to Alice using the size of the longest message and time based on an estimate of what is needed for the branching. Inferring upper bounds on the time needed for queuing is orthogonal to the work in this paper and we opt for using simple estimates. Finally, the service computes whether a majority agrees or disagrees with Alice and sends a corresponding message. We observe that no bandwidth is needed until ten votes have been received by the service. Since it cannot be determined statically when this occurs our approach reduces the traffic overhead induced compared with constant rate padding schemes as it allows scheduling of traffic on an as-needed basis, as long as the program context is public.

The main contributions of this paper are:

- We spotlight the gap in the assumptions made in the language-based information flow literature for interactive programs and the channels available for real-world, online communication.

- We introduce SELENE, a language for using channels with observable traffic information in a principled and provably secure way, thereby recovering the strong security guarantees of language-based techniques.

- We introduce a novel model that combines program and runtime behaviour in a single small-step semantics, and give a knowledge-based security condition for timingsensitive, progress-sensitive noninterference.

- We provide a progress-sensitive type system using both values of a fixed size type and values of a variable size type.

- We prove soundness of our type system, thereby obtaining a static guarantee that well-typed programs in SELENE do not leak via traffic patterns.

The remainder of this paper is structured as follows. In Section II we specify the threat model and provide the syntax and semantics of SELENE. We define attacker knowledge and give a strong security condition against traffic analysis attacks in Section III. We present the security type system for SELENE and prove it sound in Section IV. Finally, we discuss our work in Section $\mathrm{V}$ and give related work in Section VI, before we conclude in Section VII.

\section{SECURITY MODEL AND LANGUAGE}

This section presents our model and the syntax and semantics of our language.

\section{A. Security model}

SELENE is an interactive, imperative language for single threaded, interactive programs with blocking receives. The language is largely standard, apart from our message sending primitives and command sizeof for computing the size of a value. We assume a standard security lattice $\mathcal{L}$ of security levels $\ell$, with distinguished top and bottom elements, $T$ and $\perp$, lattice ordering $\sqsubseteq$, and least upper bound operation $\sqcup$. Each variable has a fixed security level that does not change during execution.

As is standard in prior work on information flow control, we focus on confidentiality at the local node. Remote nodes 
trusted at some level $\ell$ are also trusted to appropriately protect information sent to them up to level $\ell$. We further assume that remote nodes are also running SELENE programs. We model incoming traffic using lists. This modelling choice was shown equivalent to functional strategies for modelling deterministic, interactive programs by Clark and Hunt [13]. To this end, we consider an input environment $I$ mapping each channel to a (possibly empty) list of input packets and let program values be obtainable from a sequence of packets corresponding to the value. For simplicity, we identify channels by their security level.

We observe that traffic analysis attacks exploit patterns in traffic to make inferences about the secret state of a system without requiring that the adversary can read the contents of packets. We therefore make the simplifying assumption that the contents of packets are sufficiently protected against adversaries, e.g. by using encryption, but allow the adversary to observe the presence, recipient, and time of packets. We assume that packets are of fixed size, thereby transforming the question of packet size into a question of packet count.

\section{B. Threat model}

We consider interactive, distributed programs that communicate with remote network nodes. We consider an active adversary who is trusted at a security level $\ell_{a d v}$, who knows the program being run on the local node, and who knows initial secrets up to level $\ell_{a d v}$. Additionally, the adversary eavesdrops on incoming and outgoing encrypted communication of the local node, observing packet presence, timing, and the remote communication party. Communication on a channel is encrypted corresponding to the security level of the channel, and the adversary can decrypt and read packets on channels up to security level $\ell_{a d v}$. The objective of the adversary is to refine their knowledge on initial secrets.

\section{The language and program semantics}

$$
\begin{aligned}
e::= & n|s| x \mid e \oplus e \\
c::= & x=e|c ; c| \text { skip }|\operatorname{sleep}(e)| x=\operatorname{sizeof}(e) \\
& \mid \text { if } e \text { then } c \text { else } c \mid \text { while } e \text { do } c \\
& |x=\operatorname{in}(\ell)| \operatorname{schedule}(\ell, e, e) \mid \text { queue }(\ell, e)
\end{aligned}
$$

Figure 1. Syntax of the language

Figure 1 presents the syntax of our language. We explain the formal semantics and explain the nonstandard features.

We use a big-step semantics for evaluating expressions and assume these take unit time. The rules are standard and are given in Fig. 2. We let $\oplus$ range over total operations on arithmetic expressions. The values of our language are integers $n$ and strings $s$. We let Int denote the set of integers and String denote the set of strings and let Val = Int $\uplus$ String.

$$
\begin{gathered}
\frac{v \in V a l}{\langle v, m\rangle \Downarrow v} \quad \frac{m(x)=v}{\langle x, m\rangle \Downarrow v} \\
\frac{\left\langle e_{1}, m\right\rangle \Downarrow v_{1} \quad\left\langle e_{2}, m\right\rangle \Downarrow v_{2} \quad v=v_{1} \oplus v_{2}}{\left\langle e_{1} \oplus e_{2}, m\right\rangle \Downarrow v}
\end{gathered}
$$

Figure 2. Semantics for evaluating expressions

Programs are typed using fixed typing environment $\Gamma$. We write $\Gamma(x)=\sigma @ \ell$ to denote that variable $x$ has type $\sigma$ and security level $\ell$. The types of our language are int and string , where $\ell$ is the security level of the size of the string. Input packets either contain (part of) an input value, or are dummy. We write $v_{N}^{j}$ to denote the $j$ 'th of $N$ packets encoding value $v$ and let $\square$ denote dummy packets.

For evaluating program commands $c$ we use a small-step semantics transition $\left.\langle c, m, I\rangle \stackrel{t s}{\rightarrow} \alpha c^{\prime}, m^{\prime}, I^{\prime}\right\rangle$, where $m$ is a memory, $I$ is an input environment, and $\alpha$ is a program event generated by the step. Program steps take place at a time $t s$, however they do not increment time. We instead define a global semantics on top of the program semantics and let global steps increment time. We discuss the global semantics shortly. Program events can be empty, denoted by $\epsilon$, or an assignment, enqueue, scheduling, or input event as given by the following grammar:

$$
\alpha::=\epsilon|\mathrm{a}(x, v)| \mathrm{q}(\ell, v)|\mathrm{s}(\ell, n, n)| \mathrm{i}(\ell, x, v)
$$

Fig. 3 presents the stepping rules of our program operational semantics.

SizeOf: Command sizeof evaluates an expression to obtain a value and returns the number of packets needed to store that value. This is useful as the language requires the programmer to explicitly schedule the number of packets they wish to send. We assume a fixed packet size $\eta$, and assume that all integers are of fixed size, and that the size of a string is dependent on the length of the string.

In: The transitions of our small-step semantics for program commands are parametric in timestamps $t s$, allowing us to model blocking by conditioning transitions on $t s$. We model network input using primitive $x=\operatorname{in}(\ell)$. To preserve the type of variable $x$, the input primitive determines whether $m(x)$ is an integer or a string value, captured by set $A$ in rule IN, and uses this as argument for auxiliary function choose (Fig. 4), along with the packet sequence for channel $\ell$ and timestamp ts. Function choose is a partial function, modelling the potential for blocking. The choose function adds packets of the appropriate type to an accumulator used for decoding an input value, and steps over packets of other type, discarding any dummy packets. It returns a decoded value and the remaining packet sequence for the channel if successful. 
SIZEOF

$$
\text { ASSIGN }
$$

$\frac{\langle e, m\rangle \Downarrow v}{\langle x=e, m, I\rangle \stackrel{t s}{\rightarrow} \mathrm{a}(x, v)\langle\text { stop, } m[x \mapsto v], I\rangle}$

$$
\langle e, m\rangle \Downarrow v \quad n=\left\lceil\frac{\operatorname{size}(v)}{\eta}\right\rceil
$$$$
\langle x=\operatorname{sizeof}(e), m, I\rangle \stackrel{t s}{\rightarrow} \mathrm{a}(x, n)\langle\text { stop, } m[x \mapsto n], I\rangle
$$

\section{SEQ-1}

$$
\begin{aligned}
& \text { SKIP } \\
& \overline{\langle\text { skip, } m, I\rangle \stackrel{t s}{\rightarrow}_{\epsilon}\langle\text { stop, } m, I\rangle} \\
& \begin{array}{c}
\frac{\left\langle c_{1}, m, I\right\rangle \stackrel{t s}{\rightarrow}_{\alpha}\left\langle c_{1}^{\prime},\right.}{\left\langle c_{1} ; c_{2}, m, I\right\rangle}, \\
\geq 0 \quad r=t s+w \\
\rightarrow_{\epsilon}\langle\operatorname{await}(r), m, I\rangle
\end{array} \\
& \frac{\langle e, m\rangle \Downarrow w \quad w \geq 0 \quad r=t s+w}{\langle\operatorname{sleep}(e), m, I\rangle \stackrel{t s}{\longrightarrow}{ }_{\epsilon}\langle\operatorname{await}(r), m, I\rangle}
\end{aligned}
$$

IF-T

$$
\langle e, m\rangle \Downarrow v \quad v \neq 0
$$

$\left\langle\right.$ if $e$ then $c_{1}$ else $\left.c_{2}, m, I\right\rangle \stackrel{t s}{\longrightarrow}\left\langle c_{1}, m, I\right\rangle$

WHILE

SCHEDULE

$$
\frac{\left\langle e_{1}, m\right\rangle \Downarrow n \quad\left\langle e_{2}, m\right\rangle \Downarrow w \quad w \geq 0 \quad t=t s+w}{\left\langle\text { schedule }\left(\ell, e_{1}, e_{2}\right), m, I\right\rangle \stackrel{t s}{\longrightarrow}_{\mathrm{s}(\ell, n, t)}\langle\text { stop, } m, I\rangle}
$$

$$
\begin{aligned}
& \overline{\langle\text { while } e \text { do } c, m, I\rangle \stackrel{t s}{\longrightarrow}_{\epsilon}\langle\text { if } e \text { then } c \text {; while } e \text { do } c \text { else skip, } m, I\rangle} \\
& \frac{\text { IN }}{A \in\{\text { Int, String }\} \quad m(x) \in A \quad I(\ell)=\vec{p} \quad(v, \vec{q})=\operatorname{choose}(\vec{p}, A, t s,[])} \\
& \langle x=\operatorname{in}(\ell), m, I\rangle \stackrel{t s}{\rightarrow}_{\mathrm{i}(\ell, x, v)}\langle\operatorname{stop}, m[x \mapsto v], I[\ell \mapsto \vec{q}]\rangle
\end{aligned}
$$

SEQ-2

IF-E

$\left\langle\right.$ if $e$ then $c_{1}$ else $\left.c_{2}, m, I\right\rangle \stackrel{t s}{\rightarrow}\left\langle c_{2}, m, I\right\rangle$

AWAIT

$\overline{\langle\operatorname{await}(r), m, I\rangle \stackrel{t s}{\rightarrow}_{\epsilon}\langle\text { stop, } m, I\rangle}$

$\frac{\langle e, m\rangle \Downarrow v}{\langle\text { queue }(\ell, e), m, I\rangle \stackrel{t s}{\rightarrow} \mathrm{q}(\ell, v)\langle\text { stop, } m, I\rangle}$

Figure 3. Local operational semantics

Figure 4. Choose function

We let the packets be annotated with timestamps and require that all packets corresponding to a value have been received before the value can be obtained. That is, the timestamp of the final packet must be at or before the timestamp in the transition of the in command. If no value can be retrieved, the program blocks and cannot step.

We model internal input (e.g. reading files) only abstractly, by considering them bound in program variables.
Schedule and queue: The schedule command takes three arguments; a channel, a number of packets to be sent, and a delay before sending the packets. This issues a request to the runtime system. We describe the runtime system shortly. Command queue takes a channel and an expression as arguments and evaluates the expression to obtain a value. It then instructs the runtime system to add the value to a buffered output queue associated with the channel. 
Internal commands: Commands await and stop are only used internally and are therefore not part of the language syntax. Command await is reached from command sleep and blocks for a specified duration of time. Command stop denotes a final program configuration that cannot step any further.

\section{The runtime and the global semantics}

A key feature of our model is the global configuration modelling the language runtime. The runtime maintains the output queues in output environment $O$ and processes the packet schedule $\pi$. We present a small-step semantics for the global transitions in Fig. 5. We explain the interaction between the program configuration and global configuration in more detail. We let the global configuration contain a program configuration and require that the program steps whenever possible. For the sake of brevity, we let $P$ denote a program configuration $\langle c, m, I\rangle$. Program steps are done by rule GSTEP and emit a possibly empty event $\alpha$. We let the program communicate updates to the runtime through schedule and queue events. To this end, we apply update function upd (Fig. 6) to the schedule and output environment using event $\alpha$. If the program step emits a queue event $\mathrm{q}(\ell, v)$, value $v$ is split into a number of packets based on the size of the value, and the packets are added to the buffered output queue for channel $\ell$. If the program step emits a schedule event $\mathrm{s}(\ell, n, t)$, we use function rsv to reserve time in the schedule for a number of packets on a channel by recursively adding to $\operatorname{dom}(\pi)$. We assume that the schedule is never full, i.e. the function will terminate having scheduled all $n$ packets. We make the simplifying assumption that at most one packet can be sent in any single step and formally model the schedule as a partial function from timestamps to channels.

To model packets being sent, we extend the grammar for events with runtime events $\beta$. The runtime emits an empty event if the schedule is undefined for the current timestamp, otherwise we use function send defined below to obtain an event corresponding to the first packet in the scheduled channel's output queue, and an updated output environment. If no packets are queued on the channel, an empty dummy packet is generated and sent.

$$
\operatorname{send}(O, \ell) \triangleq \begin{cases}(\mathrm{o}(\ell, p), O[\ell \mapsto \vec{q}]) & \text { if } O(\ell)=p \cdot \vec{q} \\ (\mathrm{o}(\ell, \boldsymbol{\square}), O) & \text { if } O(\ell)=[]\end{cases}
$$

To combine program generated events $\alpha$ with runtime generated events $\beta$ we let global events $\gamma$ be a triple $(t s: \alpha, \beta)$, where $t s$ is the timestamp of the event. The observations an attacker makes on a run of a program are given by a trace of global events, each containing the timestamp of the step, leading to a timing-sensitive model. While a network attacker does not observe program events $\alpha$, maintaining them in global events is nevertheless useful, as it allows us to more easily reason about the exact state of a run. In Section III we define our security condition in terms of an attacker that does not observe program events, i.e., that observes all program events as the empty event $\epsilon$.
We extend the grammar as follows:

$$
\begin{aligned}
& \beta::=\epsilon \mid \circ(\ell, p) \\
& \gamma::=(t s: \alpha, \beta)
\end{aligned}
$$

The global configuration maintains clock $t s$ that is incremented for each step. If a program configuration is blocking, that is, if it cannot take a step at the current timestamp, but has not stopped with command stop, the global configuration steps by G-BLOCK, processing the runtime and incrementing the clock. Finally, G-STOP allows the global configuration to continue processing the runtime after the program configuration has reached command stop, provided there are scheduled packets left to process.

\section{SECURITY CONDITION}

In this section we present the security condition for timingsensitive, progress-sensitive noninterference.

We define our security condition using the knowledge-based approach [2]. The insight of this approach is to consider what an attacker observes during the execution of a program and define knowledge as the set of initial states that are consistent with seeing the execution up to this point. The security condition is then defined as a bound on how much the knowledge is allowed to change for each step of the execution. In this paper we do not consider declassification and we therefore require that attacker knowledge does not change with new observations.

\section{A. Auxiliary definitions}

We define attacker knowledge and timing-sensitive, progress-sensitive noninterference in terms of an equivalence relation on program configurations and the attacker observable trace emitted from a run. We give these auxiliary definitions before proceeding to define the security condition.

To denote that two memories are equivalent up to $\ell_{a d v}$ we write $m \approx_{\ell_{a d v}} m^{\prime}$ (Definition 1).

Definition 1 (Memory equivalence up to level). Two memories $m$ and $m^{\prime}$ are equivalent up to level $\ell_{a d v}$, written $m \approx_{\ell_{a d v}} m^{\prime}$, if for all $x \in \operatorname{dom}(\Gamma)$ both the following hold:

1) $\Gamma(x)=\sigma @ \ell \wedge \ell \sqsubseteq \ell_{a d v} \Longrightarrow m(x)=m^{\prime}(x)$

2) $\Gamma(x)=\operatorname{string}_{\ell^{\prime}} @ \ell \wedge \ell^{\prime} \sqsubseteq \ell_{a d v} \Longrightarrow \operatorname{size}(m(x))=$ $\operatorname{size}\left(m^{\prime}(x)\right)$

This definition captures that the values of attacker observable variables must have the same value, and that the size of the value of variables must be the same if the size is attacker observable.

We overload the notation and write $I \approx_{\ell_{a d v}} I^{\prime}$ to denote that two input environments are equivalent up to $\ell_{a d v}$ (Definition 2). The definition requires equality of incoming packets on attacker observable channels, and uses relation $\approx_{\ell}^{\text {net }}$ for high channels, requiring that these receive packets at the same timestamps. This captures an attacker that can observe the presence of incoming packets on all channels and when they arrive, but who cannot read the contents of packets on high 


$$
\begin{aligned}
& \text { G-STEP } \\
& \frac{P \stackrel{t s}{\rightarrow}{ }_{\alpha} P^{\prime} \quad\left(O^{\prime}, \pi^{\prime}\right)=\operatorname{upd}(O, \pi, \alpha) \quad\left(\beta, O^{\prime \prime}\right)= \begin{cases}\left(\epsilon, O^{\prime}\right) & \text { if } t \notin \notin \operatorname{dom}\left(\pi^{\prime}\right) \\
\operatorname{send}\left(O^{\prime}, \ell\right) & \text { if } \pi^{\prime}(t s)=\ell\end{cases} }{\left\langle P, O, \pi, t s \downarrow \rightarrow{ }_{(t s: \alpha, \beta)} \backslash P^{\prime}, O^{\prime \prime}, \pi^{\prime}, t s+1 \downarrow\right.}
\end{aligned}
$$

G-BLOCK

$$
\frac{\nexists P^{\prime}: P \stackrel{t s}{\rightarrow}{ }_{\alpha} P^{\prime} \quad P=\langle c, m, I\rangle \quad c \neq \text { stop } \quad\left(\beta, O^{\prime}\right)= \begin{cases}(\epsilon, O) & \text { if } t s \notin \operatorname{dom}(\pi) \\ \operatorname{send}(O, \ell) & \text { if } \pi(t s)=\ell\end{cases} }{\left\langle P, O, \pi, t s \downarrow \rightarrow{ }_{(t s: \epsilon, \beta)} \backslash P, O^{\prime}, \pi, t s+1 \downarrow\right.}
$$

G-STOP

$$
\frac{P=\langle\text { stop, } m, I\rangle \quad \exists t s^{\prime} \in \operatorname{dom}(\pi): t s^{\prime} \geq t s \quad\left(\beta, O^{\prime}\right)= \begin{cases}(\epsilon, O) & \text { if } t \notin \notin \operatorname{dom}(\pi) \\ \operatorname{send}(O, \ell) & \text { if } \pi(t s)=\ell\end{cases} }{\left\langle P, O, \pi, t s \downarrow \rightarrow_{(t s: \epsilon, \beta)} \backslash P, O^{\prime}, \pi, t s+1 \downarrow\right.}
$$

Figure 5. Global operational semantics

$$
\operatorname{split}(v) \triangleq \square_{N}^{1} \cdot \ldots \cdot \varpi_{N}^{N} \quad \text { where } N=\left\lceil\frac{\operatorname{size}(v)}{\eta}\right\rceil
$$

$$
\begin{aligned}
& \operatorname{rsv}(\pi, \ell, n, t) \triangleq \begin{cases}\pi_{r} & \text { if } n>0 \text { and } t \notin \operatorname{dom}(\pi) \text { and } \\
& \operatorname{rsv}(\pi[t \mapsto \ell], \ell, n-1, t+1)=\pi_{r} \\
\pi_{r} & \text { if } n>0 \text { and } t \in \operatorname{dom}(\pi) \text { and } \\
& \operatorname{rsv}(\pi, \ell, n, t+1)=\pi_{r} \\
\pi & \text { if } n \leq 0\end{cases} \\
& \operatorname{upd}(O, \pi, \alpha) \triangleq \begin{cases}\left(O^{\prime}, \pi\right) & \text { if } \alpha=\mathrm{q}(\ell, v), \operatorname{split}(v)=\vec{p}, \\
\left(O, \pi^{\prime}\right) & O \text { if } \alpha=\mathrm{s}(\ell, n, t) \text { and } \\
& \operatorname{rsv}(\pi, \ell, n, t)=\pi^{\prime}\end{cases} \\
& (O, \pi) \quad \text { otherwise }
\end{aligned}
$$

Figure 6. Runtime function

channels. We assume that incoming packets are sent by other SELENE programs and hence are of fixed size.

Definition 2 (Input environment equivalence up to level). Two input environments $I$ and $I^{\prime}$ are equivalent up to level $\ell_{a d v}$, written $I \approx_{e_{a d v}} I^{\prime}$, if

$$
\frac{\ell \sqsubseteq \ell_{a d v} \Longrightarrow I_{1}(\ell)=I_{2}(\ell)}{\ell \nsubseteq \ell_{a d v} \Longrightarrow I_{1}(\ell) \approx_{\ell_{a d v}}^{\text {net }} I_{2}(\ell)}
$$

where $\approx_{\ell_{a d v}}^{\text {net }}$ is defined by

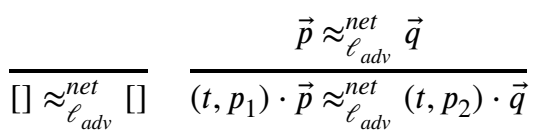

We lift equivalences to program configurations in a straight forward way in Definition 3 .

Definition 3 (Program configuration equivalence up to level). Two program configurations $\left\langle c_{1}, m_{1}, I_{1}\right\rangle$ and $\left\langle c_{2}, m_{2}, I_{2}\right\rangle$ are equivalent up to level $\ell_{a d v}$, written

$$
\left\langle c_{1}, m_{1}, I_{1}\right\rangle \approx_{\ell_{a d v}}\left\langle c_{2}, m_{2}, I_{2}\right\rangle
$$

if it holds that $c_{1}=c_{2}, m_{1} \approx_{\ell_{a d v}} m_{2}$, and $I_{1} \approx_{\ell_{a d v}} I_{2}$.

We overload the notation even further and write $O \approx_{\ell_{a d v}} O^{\prime}$ to denote that two output environments are equivalent up to $\ell_{a d v}$ (Definition (4).

Definition 4 (Output environment equivalence up to level). Two output environments $O_{1}$ and $O_{2}$ are equivalent up to level $\ell_{a d v}$, written $O_{1} \approx_{\ell_{a d v}} O_{2}$, if

$$
\frac{\ell \sqsubseteq \ell_{a d v} \Longrightarrow O_{1}(\ell)=O_{2}(\ell)}{O_{1} \approx_{\ell_{a d v}} O_{2}}
$$

Next, we define runtime event projections. Runtime event projection captures the observable parts of output events emitted by the runtime. We write $\lfloor\beta\rfloor_{\ell_{a d v}}$ to denote the projection of runtime event $\beta$ to level $\ell_{a d v}$ (Definition 5). We introduce a new event capturing the sending of packets with unobservable content. We extend the grammar for runtime events as follows:

$$
\beta::=\ldots \mid \circ(\ell,-)
$$


Runtime event $\mathrm{o}(\ell, p)$ projects to $\mathrm{o}(\ell,-)$ if the level of the channel does not flow to the level being projected to. This captures the assumption that the contents of packets can be securely hidden by cryptography, while the presence of packets and their recipient remain visible.

Definition 5 (Runtime event projection). The projection of runtime event $\beta$ to level $\ell_{a d v}$, written $\lfloor\beta\rfloor_{\ell_{a d v}}$, is defined as

$$
\begin{aligned}
\lfloor\epsilon\rfloor_{\ell_{a d v}} & =\epsilon \\
\lfloor\mathrm{o}(\ell, p)\rfloor_{e_{a d v}} & = \begin{cases}\mathrm{o}(\ell, p) & \text { if } \ell \sqsubseteq \ell_{a d v} \\
\mathrm{o}(\ell,-) & \text { if } \ell \nsubseteq \ell_{a d v}\end{cases}
\end{aligned}
$$

We write $\tau \uparrow \ell_{a d v}$ to denote the filtering of trace $\tau$ to what is visible at level $\ell_{a d v}$ (Definition [6). We use this to restrict attacker knowledge to the steps where observable events are emitted. This allows us to consider secure programs such as if $\mathrm{h}$ then sleep(10) else skip, as no output occurs after branching on the secret. We let trace filtering fix the empty event $\epsilon$ as the program event component, thereby removing all program events emitted. This captures a network attacker, that only obtains new information by observing packets being sent.

Definition 6 (Trace filtering). The filtering of a trace $\tau$ to level $\ell_{a d v}$, written $\tau \uparrow \ell_{a d v}$, is defined as

$$
\begin{gathered}
\epsilon \uparrow \ell_{a d v}=\epsilon \\
\left(\tau^{\prime} \cdot(t s: \alpha, \beta)\right) \uparrow \ell_{a d v}= \\
\begin{cases}\tau^{\prime} \uparrow \ell_{a d v} \cdot\left(t s: \epsilon,\lfloor\beta\rfloor_{\ell_{a d v}}\right) & \text { if }\lfloor\beta\rfloor_{\ell_{a d v}} \neq \epsilon \\
\tau^{\prime}\left\lceil\ell_{a d v}\right. & \text { otherwise }\end{cases}
\end{gathered}
$$

As two final building blocks, we let $O_{\text {init }}$ denote the initially empty output environment and let $\pi_{i n i t}$ denote the initially empty schedule. That is,

$$
\begin{aligned}
\forall \ell \in \mathcal{L}: O_{\text {init }}(\ell) & =[] \\
\operatorname{dom}\left(\pi_{\text {init }}\right) & =\emptyset
\end{aligned}
$$

\section{B. Knowledge and noninterference}

Using the above we define the knowledge of an attacker at level $\ell_{a d v}$ after observing trace $\tau$. This definition follows the style of other knowledge-based security conditions [5], and intuitively states that an attacker may not refine their knowledge by observing new events.

Definition 7 (Attacker knowledge). Given a program configuration $P$, such that $\left\langle P, O_{\text {init }}, \pi_{\text {init }}, 0 \searrow \rightarrow_{\tau}^{*}\left\langle P^{\prime}, O^{\prime}, \pi^{\prime}, t s^{\prime} \backslash\right.\right.$, the attacker knowledge at level $\ell_{a d v}$ is the set of program configurations $P_{2}$, that are consistent with observations at that level:

$$
\begin{aligned}
& k\left(P, \tau, \ell_{a d v}\right) \triangleq \\
& \quad\left\{P_{2} \mid P \approx_{\ell_{a d v}} P_{2} \wedge\right. \\
& \quad \backslash P_{2}, O_{\text {init }}, \pi_{\text {init }}, 0 \triangleright \rightarrow_{\tau_{2}}^{*} \backslash P_{2}^{\prime}, O_{2}^{\prime}, \pi_{2}^{\prime}, t s_{2}^{\prime} \downarrow \wedge \\
& \quad\left(\tau \mid \ell_{a d v}\right)=\left(\tau_{2}\left\lceil\ell_{a d v}\right)\right\}
\end{aligned}
$$

Using the definition of attacker knowledge we define timingsensitive, progress-sensitive noninterference.
Definition 8 (Timing-sensitive, progress-sensitive noninterference). Given program configuration $P$ such that

$$
\triangleleft P, O_{\text {init }}, \pi_{\text {init }}, 0 \triangleright \rightarrow_{\tau \cdot \gamma}^{*} \backslash P^{\prime}, O^{\prime}, \pi^{\prime}, t s^{\prime} \searrow
$$

the run satisfies timing-sensitive, progress-sensitive noninterference if for all $\ell_{a d v}$ it holds that

$$
k\left(P, \tau \cdot \gamma, \ell_{a d v}\right) \supseteq k\left(P, \tau, \ell_{a d v}\right)
$$

This definition states that memories and input environments considered possible before observing global event $\gamma$ are also considered possible after observing $\gamma$, capturing that the adversary learns nothing by observing the event. To demonstrate the security condition, we rewrite Program 3 from Section [ in the syntax of SELENE. We consider one run where secret variable $\mathrm{h}$ is set to 1 and another where it is set to 0 , and assume that $n+1$ packets are needed to send a string of length $n$.

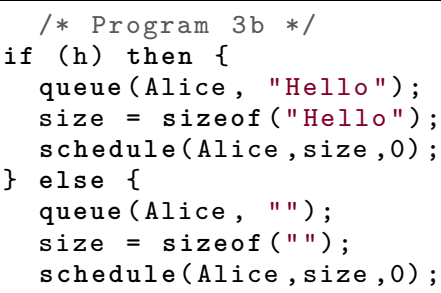

In the first run, 6 packets are scheduled and sent on channel Alice in order to send the string. In the second run, only a single packet is scheduled and sent. As the presence of every packet is observable to the attacker, they can distinguish between the two runs when a second packet is sent, hence violating Definition 8

\section{ENFORCEMENT}

In this section we present the security type system for SELENE and prove that all runs of well-typed programs satisfy timing-sensitive, progress-sensitive noninterference. Despite considering an attacker that observes only network events, timing-sensitivity makes the attacker quite strong. We settle for a rather restrictive type system that is secure against a stronger, internal attacker and we use this to show security for a network attacker. Previous work on noninterference for interactive programs by O'Neill et al. [27] achieves a more permissive type system by assuming a timing-insensitive attacker, disallowing high-loops, and assuming that new input is always available, thereby ruling out high-divergence of programs. Unfortunately, timing-insensitive attacker models are insufficient for external attackers, such as the network attacker we consider, as we cannot restrict an attacker's access to timing channels.

As our security condition is timing-sensitive and progresssensitive, our typing judgements are progress-sensitive. They are of the form

$$
\Gamma, p c \vdash c: p c^{\prime}
$$

where $p c$ is the program counter before typing the command and $p c^{\prime}$ is the program counter after. As we do not consider $p c$ declassification in this paper, the program counter never goes 
down. This leads to so-called $p c$-creep, which significantly restricts the programs that can be written in the language. We leave it to future work to explore language primitives for mitigating this and to investigate the security impact of $p c$ declassification on traffic analysis attacks.

\section{A. Type system}

The definitions of memory equivalence and event projection in Section III implicitly require a well-formedness condition on security types of variables in $\Gamma$. We now formally state this condition and assume for the rest of the paper that all variables in $\Gamma$ have well-formed security types.

$$
\overline{\vdash_{\mathrm{wf}} \text { int@e }} \quad \frac{\ell^{\prime} \sqsubseteq \ell}{\vdash_{\mathrm{wf}} \text { string }_{\ell^{\prime}} @ \ell}
$$

The intuition behind the well-formedness condition is that knowing a value implies knowing its size, but not the other way around. Next, we define subtyping relation $<$ : The relation is straight forward, using lattice ordering $\sqsubseteq$ on size levels as a condition on strings.

$$
\overline{\text { int }<\text { int }} \quad \frac{\ell_{1} \sqsubseteq \ell_{2}}{\operatorname{string}_{\ell_{1}}<: \operatorname{string}_{\ell_{2}}}
$$

We write $\sigma \nearrow \ell$ to denote raising type $\sigma$ to at least level $\ell$. This is used to account for $p c$-taint when assigning strings, to prevent string size from leaking secrets. This allows us to concisely write the conditions for the typing rules.

$$
\sigma \nearrow \ell \triangleq \begin{cases}\text { int } & \text { if } \sigma=\text { int } \\ \operatorname{string}_{\left(\ell \cup \ell^{\prime}\right)} & \text { if } \sigma=\text { string }_{\ell^{\prime}}\end{cases}
$$

The type system for expressions is presented in Fig. 7 The rules are standard, except for the rule for string expressions. This rule follows from the well-formedness condition on security types, and intuitively as the string appears in the program text, hence the size of the string is public.

\begin{tabular}{c}
$\frac{n \in \text { Int }}{\Gamma \vdash n: \text { int } @ \perp} \frac{s \in \text { String }}{\Gamma \vdash s: \text { string }_{\perp} \perp \perp} \frac{}{\Gamma \vdash x: \Gamma(x)}$ \\
$\frac{\Gamma \vdash e_{1}: \text { int@ } \ell_{1} \quad \Gamma \vdash e_{2}: \text { int } @ \ell_{2}}{\Gamma \vdash e_{1} \oplus e_{2}: \text { int@ } \ell_{1} \sqcup \ell_{2}}$ \\
\hline
\end{tabular}

Figure 7. Type system for expressions

We present our type system for commands in Fig. 8 and explain the nonstandard rules.

SizeOf: Rule T-SizEOF expresses that the size of an integer value may be assigned to a variable conditioned only by $p c$. This is intuitively safe as integers have fixed size. The size of a string value may be assigned to a variable if the variable is at least as secret as the least upper bound of $p c$ and the size level of the string.
In: Rule T-IN is similar to input rules in previous work. We require that the level of $p c$ flows to the level of the channel $\ell$. This is to preserve low equivalence of the input environment during steps under high $p c$. As a non-standard condition, the rule uses type raising and the subtyping relation to require $\sigma_{x} \nearrow \ell<: \sigma_{x}$. For $\sigma_{x}=$ int, this condition is trivially satisfied by the definitions. For $\sigma_{x}=$ string $_{\ell^{\prime}}$, this condition corresponds to the condition $\ell \sqsubseteq \ell^{\prime}$. Intuitively, we consider both the size and the value of a received strings to be as secret as the level of the channel.

Schedule and queue: Rule T-SCHEDULE restricts schedule commands to public $p c$ and restricts the integer arguments to also be public. These conditions are natural, as we assume that traffic is publicly observable. As a consequence of progresssensitive typing, a schedule command cannot occur after the $p c$ has been tainted. The queuing of messages is by rule T-QUEUE less restrictive, and is akin to rules for sending in previous information flow literature.

We note in particular that Programs 5, 6, and 7 from Section Iare typeable by the typing rules, while the rewritten Program $3 \mathrm{~b}$ from Section III is not as it performs scheduling after branching on a high variable.

\section{B. Program configuration}

We show soundness of our security type system in a number of steps. We show that the type system of SELENE is secure against a stronger, internal attacker and show that this implies security against an external attacker. This is intuitively safe as the external attacker has weaker observational power.

We begin by defining well-formedness conditions on memories (Definition 9) and program configurations (Definition 10). These conditions are standard. We define memory $m$ to be well-formed with respect to typing environment $\Gamma$ in the straight forward way.

Definition 9 (Well-formedness of memory w.r.t. a typing environment). Given a memory $m$ and a typing environment $\Gamma$, we say that $m$ is well-formed w.r.t. $\Gamma$ if for all $x \in \operatorname{dom}(\Gamma)$ we have

(1) $m(x) \in$ Int $\Longrightarrow \Gamma(x)=\operatorname{int} @ \ell$

(2) $m(x) \in$ String $\Longrightarrow \Gamma(x)=$ string $_{\ell^{\prime}} @ \ell$

We define program configuration $\langle c, m, I\rangle$ to be well formed with respect to a typing environment $\Gamma$ and program counters $p c, p c^{\prime}$ if $c$ is stop of if $c$ is typable, and if $m$ is well-formed with respect to $\Gamma$.

Definition 10 (Well-formedness of program configurations). We say that program configuration $\langle c, m, I\rangle$ is well-formed w.r.t. a typing environment $\Gamma$ and levels $p c, p c^{\prime}$ when both the following hold:

(1) either $c$ is stop or the program is well-typed, i.e., $\Gamma, p c \vdash$ $c: p c^{\prime}$

(2) $m$ is well-formed w.r.t. $\Gamma$

Steps of the program preserve well-formedness by Lemma 1 The proof can be found in the Appendix. 


$$
\begin{aligned}
& \text { T-ASSIGN } \\
& \Gamma \vdash e: \sigma_{e} @ \ell_{e} \quad \sigma_{e} \nearrow p c<: \sigma_{x} \\
& \frac{\Gamma(x)=\sigma_{x} @ \ell_{x} \quad \ell_{e} \sqcup p c \sqsubseteq \ell_{x}}{\Gamma, p c \vdash x=e: p c} \\
& \overline{\Gamma, p c \vdash \operatorname{skip}: p c} \\
& \text { T-SIZEOF } \\
& \frac{\Gamma \vdash x: \operatorname{int} @ \ell_{x} \quad \Gamma \vdash e: \sigma_{e} @ \ell_{e} \quad p c \sqsubseteq \ell_{x} \quad \sigma_{e}=\operatorname{string}_{\ell^{\prime}} \Longrightarrow \ell^{\prime} \sqsubseteq \ell_{x}}{\Gamma, p c \vdash x=\operatorname{sizeof}(e): p c} \\
& \overline{\Gamma, p c \vdash \operatorname{await}(r): p c} \\
& \text { T-IF } \\
& \text { T-SEQ } \\
& \frac{\Gamma, p c \vdash c_{1}: p c^{\prime} \quad \Gamma, p c^{\prime} \vdash c_{2}: p c^{\prime \prime}}{\Gamma, p c \vdash c_{1} ; c_{2}: p c^{\prime \prime}} \\
& \frac{\Gamma \vdash e: \text { int@ } \ell \quad \Gamma, p c \sqcup \ell \vdash c_{1}: p c^{\prime} \quad \Gamma, p c \sqcup \ell \vdash c_{2}: p c^{\prime \prime}}{\Gamma, p c \vdash \text { if } e \text { then } c_{1} \text { else } c_{2}: p c^{\prime} \sqcup p c^{\prime \prime}} \\
& \frac{\Gamma \vdash e: \text { int } @ \ell}{\Gamma, p c \vdash \operatorname{sleep}(e): p c \sqcup \ell} \\
& \frac{\Gamma \vdash x: \sigma_{x} @ \ell_{x} \quad p c \sqsubseteq \ell \quad \sigma_{x} \nearrow \ell<: \sigma_{x} \quad \ell \sqsubseteq \ell_{x}}{\Gamma, p c \vdash x=\operatorname{in}(\ell): \ell} \\
& \Gamma, p c \vdash \text { while } e \text { do } c: p c^{\prime}
\end{aligned}
$$

\begin{tabular}{|c|c|c|}
\hline $\begin{array}{l}\text { T-SCHEDULE } \\
p c=\perp\end{array}$ & $\Gamma \vdash e_{1}:$ int $@ \perp$ & $\Gamma \vdash e_{2}:$ int $@ \perp$ \\
\hline
\end{tabular}

$\Gamma \vdash e:$ int@e $\quad \Gamma, p c \sqcup \ell \vdash c: p c^{\prime}$

Figure 8. Type system for commands

Lemma 1 (Preservation of well-formedness). Let $\Gamma$ be a typing environment, $p c, p c^{\prime}$ be two levels, and $\langle c, m, I\rangle$ be a program configuration, such that the $\langle c, m, I\rangle$ is well-formed w.r.t. $\Gamma$, $p c$, and $p c^{\prime}$. Suppose this configuration takes a step

$$
\langle c, m, I\rangle \stackrel{t s}{\longrightarrow}_{\alpha}\left\langle c^{\prime}, m^{\prime}, I^{\prime}\right\rangle
$$

Then there exists $p c^{\prime \prime}$ such that $p c \sqsubseteq p c^{\prime \prime} \sqsubseteq p c^{\prime}$ and such that the resulting program configuration $\left\langle c^{\prime}, m^{\prime}, I^{\prime}\right\rangle$ is well-formed w.r.t. $\Gamma, p c^{\prime \prime}$, and $p c^{\prime}$.

To reason about what an internal attacker learns from observing a run, we define projection of program events $\alpha$ to level $\ell_{a d v}$ (Definition 11) capturing the attacker observable changes to the internal state of the system. As our model distinguishes between the secrecy levels of the size of a string and its value, we extend the grammar for program events with an event capturing that a string of size $s$ was assigned to variable $x$.

$$
\alpha::=\ldots|| a \mid(x, s)
$$

As program event projection is similar to runtime event projection, we use similar notation, but annotate with a bullet to signify that these relate to internal state.
Definition 11 (Program event projection). The projection of program event $\alpha$ to level $\ell_{a d v}$, written $\lfloor\alpha\rfloor_{\ell_{a d v}}^{\bullet}$, is defined as

$$
\begin{aligned}
& \lfloor\epsilon\rfloor_{\ell_{a d v}}^{\bullet}=\epsilon \\
& \lfloor\mathrm{s}(\ell, n, w)\rfloor_{\ell_{a d v}}^{\bullet}=\mathrm{s}(\ell, n, w) \\
& \lfloor\mathrm{a}(x, v)\rfloor_{\ell_{a d v}}^{\bullet}= \begin{cases}\mathrm{a}(x, v) & \text { if } \Gamma(x)=\sigma @ \ell \text { s.t. } \ell \sqsubseteq \ell_{a d v} \\
|\mathrm{a}|(x, s) & \text { if } \Gamma(x)=\text { string }_{\ell^{\prime}} @ \ell \\
& \text { s.t. } \ell \nsubseteq \ell_{a d v} \wedge \ell^{\prime} \sqsubseteq \ell_{a d v} \\
& \wedge \operatorname{siz} e(v)=s \\
\epsilon & \text { otherwise }\end{cases} \\
& \lfloor\mathrm{q}(\ell, v)\rfloor_{\ell_{a d v}}^{\bullet}= \begin{cases}\mathrm{q}(\ell, v) & \text { if } \ell \sqsubseteq \ell_{a d v} \\
\epsilon & \text { if } \ell \nsubseteq \ell_{a d v}\end{cases} \\
& \lfloor\mathrm{i}(\ell, x, v)\rfloor_{\ell_{a d v}}^{\bullet}= \begin{cases}\mathrm{i}(\ell, x, v) & \text { if } \ell \sqsubseteq \ell_{a d v} \\
\epsilon & \text { if } \ell \nsubseteq \ell_{a d v}\end{cases}
\end{aligned}
$$

We define internal trace filtering using program event projection in the straight forward way. We again filter out global events where no observable program or runtime events are emitted to prevent the attacker from observing termination. 
Definition 12 (Internal trace filtering). The internal filtering of a trace $\tau$ at level $\ell_{a d v}$, written $\tau 卜^{\bullet} \ell_{a d v}$, is defined as

$$
\begin{gathered}
\epsilon \uparrow^{\bullet} e_{a d v}=\epsilon \\
\left(\tau^{\prime} \cdot(t s: \alpha, \beta)\right) \uparrow^{\bullet} e_{a d v}= \\
\begin{cases}\tau^{\prime} \Gamma^{\bullet} \ell_{a d v} \cdot\left(t s:\lfloor\alpha\rfloor_{\ell_{a d v}}^{\bullet}\lfloor\beta\rfloor_{\ell_{a d v}}\right) & \text { if }\lfloor\alpha\rfloor_{\ell_{a d v}}^{\bullet} \neq \epsilon \\
\tau^{\prime} \Gamma^{\bullet} \ell_{a d v} & \text { or }\lfloor\beta\rfloor_{\text {adv }} \neq \epsilon\end{cases}
\end{gathered}
$$

Using internal trace filtering, we define internal knowledge. This definition mirrors attacker knowledge, except for using internal trace filtering, thereby giving additional power to the attacker.

Definition 13 (Internal knowledge). Given a program configuration $P$, such that $\left\langle P, O_{\text {init }}, \pi_{\text {init }}, 0 \triangleright \rightarrow_{\tau}^{*}\left\langle P^{\prime}, O^{\prime}, \pi^{\prime}, t s^{\prime}\right\rangle\right.$, internal knowledge at level $\ell_{a d v}$ is the set of program configurations $P_{2}$, that are consistent with observations at that level:

$$
\begin{aligned}
& k^{\bullet}\left(P, \tau, \ell_{a d v}\right) \triangleq \\
& \quad\left\{P_{2} \mid P \approx_{\ell_{a d v}} P_{2} \wedge\right. \\
& \quad\left\langle P_{2}, O_{\text {init }}, \pi_{\text {init }}, 0 \triangleright \rightarrow_{\tau_{2}}^{*} \backslash P_{2}^{\prime}, O_{2}^{\prime}, \pi_{2}^{\prime}, t s_{2}^{\prime} \downarrow \wedge\right. \\
& \left.\quad\left(\tau \vdash^{\bullet} \ell_{a d v}\right)=\left(\left.\tau_{2}\right|^{\bullet} \ell_{a d v}\right)\right\}
\end{aligned}
$$

Lemma 2 captures that an internal attacker is indeed stronger than an external attacker by giving internal knowledge as a lower bound on attacker knowledge. This lemma allows us to relate the result we obtain for an internal attacker to the external attacker we consider in our threat model, thereby enabling us to show the type system of SELENE sound with respect to Definition 8 We refer to the Appendix for the proof.

Lemma 2 (Internal knowledge refines external knowledge). For any program configuration $P$, trace $\tau$, and level $\ell_{a d v}$, the knowledge of an external attacker is less precise than the knowledge of an internal attacker. That is,

$$
k\left(P, \tau, \ell_{a d v}\right) \supseteq k^{\bullet}\left(P, \tau, \ell_{a d v}\right)
$$

In defining the knowledge of a network attacker in Section [III] we defined equivalence of input environments (Definition 2. by relating only environments whose high channels receive packets at the same timestamps. However, while the network attacker observes incoming packets, they do not observe if or when the packets are consumed by the program internally. For this reason, program steps do not need to preserve equivalence by Definition 2 To relate input environments internally we define internal equivalence (Definition 14) as equality of packet sequences on attacker observable channels.

Definition 14 (Internal input environment equivalence up to level). Two input environments $I$ and $I^{\prime}$ are internally equivalent up to level $\ell_{a d v}$, written $I \approx_{\ell_{a d v}}^{\bullet} I^{\prime}$, if

$$
\frac{\ell \sqsubseteq \ell_{a d v} \Longrightarrow I_{1}(\ell)=I_{2}(\ell)}{I_{1} \approx_{\ell_{a d v}}^{\bullet} I_{2}}
$$

Definition 14 is strictly weaker than Definition 2 which we state as Lemma 3
Lemma 3 (Network input equivalence implies internal input equivalence). For any input environments $I_{1}, I_{2}$, equivalence by Definition 2 implies equivalence by Definition 14 That is,

$$
I_{1} \approx_{\ell_{a d v}} I_{2} \Longrightarrow I_{1} \approx_{\ell_{a d v}}^{\bullet} I_{2}
$$

Proof: Immediate from the definitions.

We now present our noninterference lemma for program configurations (Lemma 44). It says that given a level $\ell_{a d v}$ and a program configuration that takes a step emitting some event $\alpha$, then all configurations equivalent at $\ell_{a d v}$ either take a step, emitting an equivalent event $\alpha^{\prime}$, and are again equivalent at $\ell_{a d v}$; or the configuration can be typed with a high $p c$ and has no observable effects at level $\ell_{a d v}$.

Lemma 4 (Program step noninterference). Given a level $\ell_{a d v}$ and a command $c$ and $\Gamma, p c, p c^{\prime}$ such that $c$ is well-formed w.r.t $\Gamma, p c, p c^{\prime}$, if

$$
\left\langle c, m_{1}, I_{1}\right\rangle \stackrel{t s}{\rightarrow}_{\alpha_{1}}\left\langle c^{\prime}, m_{1}^{\prime}, I_{1}^{\prime}\right\rangle
$$

then for any memory $m_{2}$ such that $m_{1} \approx_{\ell_{a d v}} m_{2}$ and input environment $I_{2}$ such that $I_{1} \approx_{\ell_{a d v}}^{\bullet} I_{2}$, we have that one of the following holds

1) either $\left\langle c, m_{2}, I_{2}\right\rangle \stackrel{t s}{\rightarrow} \alpha_{2}\left\langle c^{\prime}, m_{2}^{\prime}, I_{2}^{\prime}\right\rangle$ such that $\left\lfloor\alpha_{1}\right\rfloor_{\ell_{a d v}}^{\bullet}=$ $\left\lfloor\alpha_{2}\right\rfloor_{\ell_{a d v}}^{\bullet}$ and $m_{1}^{\prime} \approx_{\ell_{a d v}} m_{2}^{\prime}$ and $I_{1}^{\prime} \approx_{\ell_{a d v}}^{\bullet} I_{2}^{\prime}$.

2) or $\left\lfloor\alpha_{1}\right\rfloor_{\ell_{a d v}}^{\bullet}=\epsilon$ and there is $p c^{\prime \prime}$ such that $p c^{\prime \prime} \nsubseteq \ell_{a d v}$ and $\Gamma, p c^{\prime \prime} \vdash c: p c^{\prime}$ and $m_{1} \approx_{\ell_{a d v}} m_{1}^{\prime}$ and $I_{1} \approx_{\ell_{a d v}}^{\bullet} I_{1}^{\prime}$

We refer the interested reader to the Appendix for the proof and supporting lemmas.

\section{Global configuration}

We take the next step towards showing soundness of the type system by using Lemma 4 to show single-step noninterference of global configurations. Lemma [5 tells us that for any level $\ell_{a d v}$ and any two equivalent runs, if one run takes a step producing global event $\gamma$, then the other run also takes an equivalent step, or the two runs diverge with high $p c$. We do not require that all events emitted after divergence are unobservable to the adversary. Instead, we allow runtime events to still be observable. This is intuitively safe as the runtime behaviour is fully determined by the program events, and no observable program events may be emitted after divergence. To simplify reasoning for divergent runs, we use a configuration with command stop in the second condition. This configuration serves as an anchor, allowing us to reason about the observable behaviour of any two equivalent configurations by showing they behave the same as the stopconfiguration. To satisfy this condition, the configuration must produce no observable program event or changes to memory or input environment, and produces output if and only if the stop-configuration produces equivalent output.

Lemma 5 (Global step noninterference). Given a level $\ell_{a d v}$ and a command $c$ and $\Gamma, p c, p c^{\prime}$ such that $c$ is well-formed w.r.t $\Gamma, p c, p c^{\prime}$, if

$$
\left.\triangleleft\left\langle c, m_{1}, I_{1}\right\rangle, O_{1}, \pi, t s \downarrow \rightarrow_{\left(t s: \alpha_{1}, \beta_{1}\right)} \backslash\left\langle c^{\prime}, m_{1}^{\prime}, I_{1}^{\prime}\right\rangle, O_{1}^{\prime}, \pi^{\prime}, t s^{\prime}\right\rangle
$$


then for any memory $m_{2}$ such that $m_{1} \approx_{\ell_{a d v}} m_{2}$; input environment $I_{2}$ such that $I_{1} \approx_{\ell_{q d v}}^{\bullet} I_{2}$; and output environment $\mathrm{O}_{2}$ such that $\mathrm{O}_{1} \approx_{\ell_{a d v}} \mathrm{O}_{2}$, we have that one of the following holds

\section{1) either}

$$
\left.\left.\checkmark\left\langle c, m_{2}, I_{2}\right\rangle, O_{2}, \pi, t s\right\rangle \rightarrow{ }_{\left(t s: \alpha_{2}, \beta_{2}\right)} \backslash\left\langle c^{\prime}, m_{2}^{\prime}, I_{2}^{\prime}\right\rangle, O_{2}^{\prime}, \pi^{\prime}, t s^{\prime}\right\rangle
$$

such that each of the following hold
a) $\left\lfloor\alpha_{1}\right\rfloor_{\ell_{a d v}}^{\bullet}=\left\lfloor\alpha_{2}\right\rfloor_{\ell_{a d v}}^{\bullet}$
b) $\left\lfloor\beta_{1}\right\rfloor_{\ell_{a d v}}=\left\lfloor\beta_{2}\right\rfloor_{\ell_{a d v}}$
c) $m_{1}^{\prime} \approx_{\ell_{a d v}} m_{2}^{\prime}$
d) $I_{1}^{\prime} \approx_{\ell_{a d v}}^{\bullet^{a d v}} I_{2}^{\prime}$
e) $O_{1}^{\prime} \approx_{\ell_{a d v}} O_{2}^{\prime}$

2) or there is $p c^{\prime \prime}$ such that $p c^{\prime \prime} \nsubseteq \ell_{a d v}$ and $c$ is well-formed w.r.t $\Gamma, p c^{\prime \prime}, p c^{\prime}$, and such that each of the following hold

a) $\left\lfloor\alpha_{1}\right\rfloor_{\ell_{a d v}}^{\bullet}=\epsilon$

b) $m_{1} \approx_{\ell_{a d v}} m_{1}^{\prime}$

c) $I_{1} \approx_{\ell_{a d v}}^{\bullet} I_{1}^{\prime}$

d) $\pi=\pi^{\prime}$

e) $\beta_{1} \neq \epsilon$ if and only if there are $\beta_{2}$ and $O_{2}^{\prime}$ such that

$$
\begin{aligned}
& \left\lfloor\beta_{1}\right\rfloor_{\ell_{a d v}}=\left\lfloor\beta_{2}\right\rfloor_{\ell_{a d v}}, O_{1}^{\prime} \approx_{\ell_{a d v}} O_{2}^{\prime} \text {, and } \\
& \left.\qquad\left\langle\text { stop, } m_{2}, I_{2}\right\rangle, O_{2}, \pi, t s\right\rangle \\
& \left.\quad \rightarrow{ }_{\left(t s: \epsilon, \beta_{2}\right)} \backslash\left\langle\text { stop, } m_{2}, I_{2}\right\rangle, O_{2}^{\prime}, \pi, t s^{\prime}\right\rangle
\end{aligned}
$$

We again refer to the Appendix for the proof.

\section{Soundness of security type system}

Using the above results, we are now ready to state our soundness theorem (Theorem 1). It says that any well-typed program satisfies timing-sensitive, progress-sensitive noninterference (Definition 8).

Theorem 1 (Soundness). Given a typing environment $\Gamma$, two levels $p c, p c^{\prime}$, and a program configuration $P$ that is well-formed w.r.t $\Gamma, p c, p c^{\prime}$, the run $\left\langle P, O_{\text {init }}, \pi_{\text {init }}, 0\right\rangle \rightarrow_{\tau}^{*}$ $\left.\checkmark P^{\prime}, O^{\prime}, \pi^{\prime}, t s^{\prime}\right\rangle$ satisfies Definition 8

The proof is by induction in the number of steps and uses Lemma 5 to infer that if the run emits an event observable by an internal attacker, then all runs of equivalent program configurations must as well, hence the internal attacker does not learn anything. Using Lemma 2 we conclude that the network attacker equally does not learn anything. The full proof of Theorem 1 is omitted here and can be found in the Appendix.

As noted in Section IV Programs 5, 6, and 7 from Section I are typeable by the typing rules and hence by Theorem 1 we obtain a proof that they satisfy Definition 8 and do not leak by their output behaviour.

\section{DISCUSSION}

\section{A. Publicly observable traffic}

By our chosen strategy and the assumption that network activity can be eavesdropped, we arrive at a number of restrictions on how output channels can be used. Our strategy does not permit the scheduling of new messages after the program counter has been raised. Nevertheless, Program 7 in Section I] demonstrates how dynamic scheduling is possible after receiving input, provided the input is received on a non-secret channel. This allows lower bandwidth overheads compared with constant rate padding schemes, by only scheduling traffic when needed. While it is unsurprising that it is safe to only schedule as needed while in a low context, this intuitive fact is difficult to prove correct without a principled, formal model like we present in this paper.

\section{B. Limitations and future work}

In this paper we have opted for simple and explicit packet scheduling via programmer written commands, allowing us to use the IFC system to prevent leaks from both message contents and message presence. Our model and the primitives presented are not intended as a full solution for preventing traffic analysis attacks, but rather aim to bring attention to an as yet unsolved problem, and serve as a step towards providing practical and provably safe usage of channels susceptible to eavesdropping. A significant limitation of our model is that the progress-sensitive nature of the type system makes composition of programs difficult. A pc-declassification mechanism would alleviate this issue, but the security impact of allowing such mechanism must be fully understood. To focus our model, we have deliberately not included a $p c$-declassification primitive in the language.

Other strategies for setting up packet schedules may be viable. In particular, we note that patterns in publicly observable input traffic may be used when deciding the shape of output traffic. However, we note that for receives with public blocking behaviour, the employed strategy should not incur significant overheads or hinder the ability to reply. As such, static preprocessing of a target program to determine a packet schedule is not viable.

\section{RELATED WORK}

Sabelfeld and Mantel [30] also consider the problem of sending secret messages over publicly observable channels as part of a distributed program. They consider concurrent programs that communicate over low channels that are fully observable; encrypted channels where the number of messages is observable, but size and contents is not; and high channels where both message presence and contents are secret. They define a timing-sensitive security definition using strong lowbisimulation, requiring that the number of encrypted messages sent is the same between any two related runs in lockstep. The channels they consider model communication with specific endpoints at nodes - rather than with nodes themselves - and they do not contain dummy messages. Consequently, the blocking behaviour of receives on encrypted channels is public, and encrypted channels in their work do not correspond with non-public channels of our work whose blocking behaviour is non-public. The authors discuss the practical implications of different communication primitives. They argue that receives on channels that exhibit secret blocking behaviour is not 
secure and non-blocking receives should be used instead, while receives on channels that exhibit public blocking behaviour should use blocking receives to prevent busy waiting.

Zhang et al. [36] propose a general language-based mechanism for controlling timing channels based on the idea of predictive migitation [6]. While both their approach and ours rely on the idea of scheduling observable events, they are orthogonal. A distinguishing property of the predictive mitigation is that because of its generality the only allowed modification to program semantics is delaying of the messages. In contrast, our approach - where we focus on the network attacker - allows us to use dummy messages, preventing the delays caused by mispredictions.

As noted in Section [ a a $p c$-declassification mechanism could be used to alleviate some of the restrictions imposed by SELENE's progress-sensitive type system. Bay and Askarov [9] give a formal condition on how much attacker knowledge is allowed to change as a result of $p c$-declassification by bounding it using the so-called progress-knowledge. We leave adapting their approach to SELENE as future work. Vassena et al. [33] propose a dynamic language-level IFC system that supports deterministic parallel thread execution. Such a system could retain a public context thread, potentially mitigating the need for explicit $p c$-declassification.

Oblivious programming languages such as ObliVM [24] and Obliv-C [35] allow programmers to write protocols for secure computations, where multiple parties can perform computation collaboratively without revealing their input via produced trace, e.g. instructions, memory accesses, and values of public variables. To achieve security, such languages commonly simulate the execution of non-chosen branches in conditional statements and publicly bound and pad the number of loop iterations and the number of bits needed to represent secret values. While the goal of oblivious programming languages overlaps with ours at a high level, care is needed for adapting the techniques to our model. Generally speaking, these languages do not allow loop guards or blocking behaviour to be nonpublic. Our model allows the size of network messages to be kept secret by sending them as a series of (potentially dummy) packets. Consequently, the blocking behaviour of the receive primitive for non-public channels in SELENE is inherently non-public. A solution used in the oblivious approach is to tag values with a public, conservative upper bound on their size. This gives weaker confidentiality, but if acceptable appears a viable solution for the problem we discuss in this paper and we leave application of oblivious programming techniques as future work.

Previous work has examined the possibility of traffic analysis attacks revealing sensitive user information and actions across various settings.

Browsers: Chen et al. [11] and Miller et al. [26] both consider the traffic patterns generated by user interactions on webpages. Miller et al. present an attack against the HTTPS deployments of industry-leading websites spanning multiple sectors. Their attack was able to identify pages within a site with high accuracy, exposing personal details including medical conditions and financial affairs. They propose a defence mechanism that pads contiguous bursts of traffic up to per-website, predefined thresholds. Their analysis shows that the proposed defence mechanism outperforms site-agnostic approaches that pad the sizes of all packets to global, nearest threshold values. Chen et al. find that the potential for traffic analysis attacks is exacerbated by design features for dynamic, reactive websites such as AJAX GUI widgets, which often generate distinctive traffic in response to user interactions.

Cherubin et al. [12] consider website fingerprinting defences at the application layer and introduce ALPaCA, a server side defence for use with Tor. ALPaCA works by transforming site content to conform to average site content, as analysed across multiple Tor sites. Their analysis shows that ALPaCA reduces website fingerprinting accuracy from $69.6 \%$ to $10 \%$.

Phones and apps: Conti et al. [15] and Wang et al. [34] both consider attacks on users of Android smartphones. Conti et al. present a machine learning assisted traffic analysis attack that infers user actions in apps with high precision and high recall, e.g., opening a profile page on Facebook or posting a message on Twitter. Wang et al. present a packet level attack on encrypted Android traffic. By collecting and analysing a small amount of wireless traffic, they are able to determine which apps smartphone users are using. Their analysis shows that apps are more susceptible to traffic analysis attacks than online services accessed over browsers, as apps tend to generate more distinct patterns of traffic.

Bahramali et al. [7] show that also instant messaging clients are susceptible to traffic analysis attacks despite using stateof-the-art encryption. They demonstrate an attack capable of identifying members and administrators of IM channels with high accuracy, using only low-cost traffic analysis techniques. They attribute this to the fact that major IM operators do not use mechanisms for obfuscating genuine traffic, arguing their reluctance is due to the performance and usability impact of deploying such techniques.

In the home: Zhang et al. [37] present an attack for inferring user activities by eavesdropping on WLAN traffic. They consider online activities such as web browsing, chatting, gaming, and watching videos. They use a hierarchical classification system based on machine learning algorithms and show that their system can distinguish different online applications with roughly $80 \%$ accuracy when given 5 seconds of traffic, and roughly $90 \%$ accuracy when given 1 minute of traffic.

Apthorpe et al. [1] consider home IoT devices and attacks inferring when a device is used, thereby revealing sensitive user information such as sleep patterns and when the user is home. They introduce stochastic traffic padding, which decreases attacker confidence by uniformly shaping upload and download traffic during user activities, and injecting equivalent traffic patterns at random times to hide when the device is in use.

\section{CONCLUSION}

In this paper we consider language-based mitigation of traffic analysis attacks. We observe four traits on messages 
sent that may leak secret information, namely presence, recipient, size, and time. This observation informed the design of SELENE, a small imperative language for interactive programs. The type system of SELENE enforces principled, provably secure communication over channels where packets are publicly observable. The key insight of the language is a novel primitive that provides programmatic control over traffic shaping thereby allowing for reduced overheads in latency and bandwidth compared with black box techniques. We give a formal, timing-sensitive, progress-sensitive security condition based on the knowledge-based approach and prove our type system sound. We believe that our model faithfully captures online communication constraints, and that our results constitute a step towards practical, secure online communication. We believe the security risks of traffic analysis attacks against confidentiality are significant and that work on language-based information flow for interactive programs must be mindful of the assumptions being made about the security of communications channels. We welcome and encourage future work to explore language-based techniques for providing strong security guarantees against traffic analysis attacks.

\section{ACKNOWLEDGEMENTS}

We thank Alix Trieu for his comments and feedback and thank the anonymous reviewers for their valuable suggestions for improving the presentation of this paper. This work was funded by the Danish Council Independent Research for the Natural Sciences (DFF/FNU, project 6108-00363).

\section{REFERENCES}

[1] N. J. Apthorpe, D. Y. Huang, D. Reisman, A. Narayanan, and N. Feamster, "Keeping the smart home private with smart(er) iot traffic shaping," CoRR, vol. abs/1812.00955, 2018. [Online]. Available: http://arxiv.org/abs/1812.00955

[2] A. Askarov and S. Chong, "Learning is change in knowledge: Knowledge-based security for dynamic policies," 2012 IEEE 25th Computer Security Foundations Symposium, pp. 308-322, 2012.

[3] A. Askarov and A. Sabelfeld, "Tight enforcement of information-release policies for dynamic languages," in 2009 22nd IEEE Computer Security Foundations Symposium, 2009, pp. 43-59.

[4] A. Askarov and A. C. Myers, "Attacker control and impact for confidentiality and integrity," Logical Methods in Computer Science, vol. 7, no. 3, 2011. [Online]. Available: https://doi.org/10.2168/LMCS-7(3:17)2011

[5] A. Askarov and A. Sabelfeld, "Gradual release: Unifying declassification, encryption and key release policies," in 2007 IEEE Symposium on Security and Privacy (SP'07). IEEE, 2007, pp. 207-221.

[6] A. Askarov, D. Zhang, and A. C. Myers, "Predictive black-box mitigation of timing channels," in Proceedings of the 17th ACM Conference on Computer and Communications Security, ser. CCS '10. New York,
NY, USA: ACM, 2010, pp. 297-307. [Online]. Available: http://doi.acm.org/10.1145/1866307.1866341

[7] A. Bahramali, A. Houmansadr, R. Soltani, D. Goeckel, and D. Towsley, "Practical traffic analysis attacks on secure messaging applications," Proceedings 2020 Network and Distributed System Security Symposium, 2020. [Online]. Available: http://dx.doi.org/10.14722/ndss.2020.24347

[8] I. Bastys, M. Balliu, T. Rezk, and A. Sabelfeld, "Clockwork: Tracking remote timing attacks," in 2020 IEEE 33 rd Computer Security Foundations Symposium (CSF), 2020, pp. 350-365.

[9] J. Bay and A. Askarov, "Reconciling progress-insensitive noninterference and declassification," in 2020 IEEE 33 rd Computer Security Foundations Symposium (CSF). IEEE, 2020, pp. 95-106.

[10] A. Bohannon, B. C. Pierce, V. Sjöberg, S. Weirich, and S. Zdancewic, "Reactive noninterference," in Proceedings of the 16th ACM Conference on Computer and Communications Security, ser. CCS '09. New York, NY, USA: Association for Computing Machinery, 2009, p. 79-90. [Online]. Available: https://doi.org/10.1145/1653662.1653673

[11] S. Chen, R. Wang, X. Wang, and K. Zhang, "Sidechannel leaks in web applications: A reality today, a challenge tomorrow," in Proceedings of the 2010 IEEE Symposium on Security and Privacy, ser. SP '10. USA: IEEE Computer Society, 2010, p. 191-206. [Online]. Available: https://doi.org/10.1109/SP.2010.20

[12] G. Cherubin, J. Hayes, and M. Juárez, "Website fingerprinting defenses at the application layer," PoPETs, vol. 2017, no. 2, pp. 186-203, 2017. [Online]. Available: https://doi.org/10.1515/popets-2017-0023

[13] D. Clark and S. Hunt, "Non-interference for deterministic interactive programs," in Formal Aspects in Security and Trust: 5th International Workshop, FAST 2008 Malaga, Spain, October 9-10, 2008 Revised Selected Papers, 04 2009, pp. 50-66.

[14] M. R. Clarkson and F. B. Schneider, "Hyperproperties," J. Comput. Secur., vol. 18, no. 6, pp. 1157-1210, Sep. 2010. [Online]. Available: http://dl.acm.org/citation.cfm?id=1891823.1891830

[15] M. Conti, L. V. Mancini, R. Spolaor, and N. V. Verde, "Analyzing android encrypted network traffic to identify user actions," IEEE Transactions on Information Forensics and Security, vol. 11, no. 1, pp. 114-125, 2016.

[16] D. Das, S. Meiser, E. Mohammadi, and A. Kate, "Anonymity trilemma: Strong anonymity, low bandwidth overhead, low latency - choose two," IACR Cryptology ePrint Archive, vol. 2017, p. 954, 2017.

[17] K. P. Dyer, S. E. Coull, T. Ristenpart, and T. Shrimpton, "Peek-a-boo, i still see you: Why efficient traffic analysis countermeasures fail," in Proceedings of the 2012 IEEE Symposium on Security and Privacy, ser. SP '12. USA: IEEE Computer Society, 2012, p. 332-346. [Online]. Available: https://doi.org/10.1109/SP.2012.28 
[18] J. Feigenbaum, A. Johnson, and P. Syverson, "Preventing active timing attacks in low-latency anonymous communication," in Privacy Enhancing Technologies. Berlin, Heidelberg: Springer Berlin Heidelberg, 07 2010, pp. 166-183.

[19] X. Fu, B. Graham, R. Bettati, W. Zhao, and D. Xuan, "Analytical and empirical analysis of countermeasures to traffic analysis attacks," in 2003 International Conference on Parallel Processing, 2003. Proceedings., 11 2003, pp. $483-492$.

[20] J. A. Goguen and J. Meseguer, "Security policies and security models," in 1982 IEEE Symposium on Security and Privacy, 1982, pp. 11-11.

[21] D. Hedin and A. Sabelfeld, "A perspective on information-flow control," in Software Safety and Security, 2012.

[22] M. Juárez, M. Imani, M. Perry, C. Díaz, and M. Wright, "WTF-PAD: toward an efficient website fingerprinting defense for tor," CoRR, vol. abs/1512.00524, 2015. [Online]. Available: http://arxiv.org/abs/1512.00524

[23] A. Kwon, M. AlSabah, D. Lazar, M. Dacier, and S. Devadas, "Circuit fingerprinting attacks: Passive deanonymization of tor hidden services," in Proceedings of the 24th USENIX Conference on Security Symposium, ser. SEC'15. Berkeley, CA, USA: USENIX Association, 2015, pp. 287-302. [Online]. Available: http://dl.acm.org/citation.cfm?id=2831143.2831162

[24] C. Liu, X. S. Wang, K. Nayak, Y. Huang, and E. Shi, "Oblivm: A programming framework for secure computation," in 2015 IEEE Symposium on Security and Privacy, 2015, pp. 359-376.

[25] H. Mantel and A. Sabelfeld, "A unifying approach to the security of distributed and multi-threaded programs," $J$. Comput. Secur., vol. 11, no. 4, p. 615-676, Jul. 2003.

[26] B. Miller, L. Huang, A. D. Joseph, and J. D. Tygar, "I know why you went to the clinic: Risks and realization of https traffic analysis," in Privacy Enhancing Technologies, E. De Cristofaro and S. J. Murdoch, Eds. Cham: Springer International Publishing, 2014, pp. 143-163.

[27] K. R. O'Neill, M. R. Clarkson, and S. Chong, "Information-flow security for interactive programs," in Proceedings of the 19th IEEE Workshop on Computer Security Foundations, ser. CSFW '06. Washington, DC, USA: IEEE Computer Society, 2006, pp. 190-201. [Online]. Available: https://doi.org/10.1109/CSFW.2006.16

[28] R. Overdorf, M. Juárez, G. Acar, R. Greenstadt, and C. Díaz, "How unique is your .onion? an analysis of the fingerprintability of tor onion services," CoRR, vol. abs/1708.08475, 2017. [Online]. Available: http://arxiv.org/abs/1708.08475

[29] A. Panchenko, F. Lanze, A. Zinnen, M. Henze, J. Pennekamp, K. Wehrle, and T. Engel, "Fingerprinting at internet scale," in Proceedings of the 23rd Internet Society (ISOC) Network and Distributed System Security Symposium (NDSS 2016), 2015.
[30] A. Sabelfeld and H. Mantel, "Static confidentiality enforcement for distributed programs," in Static Analysis, M. V. Hermenegildo and G. Puebla, Eds. Berlin, Heidelberg: Springer Berlin Heidelberg, 2002, pp. 376394.

[31] D. Schoepe and A. Sabelfeld, "Understanding and enforcing opacity," in Proceedings of the 2015 IEEE 28th Computer Security Foundations Symposium, ser. CSF '15. Washington, DC, USA: IEEE Computer Society, 2015, pp. 539-553. [Online]. Available: https://doi.org/10.1109/CSF.2015.41

[32] S. Siby, M. Juárez, N. Vallina-Rodriguez, and C. Troncoso, "Dns privacy not so private: the traffic analysis perspective," 2018.

[33] M. Vassena, G. Soeller, P. Amidon, M. Chan, J. Renner, and D. Stefan, "Foundations for parallel information flow control runtime systems," in Principles of Security and Trust, F. Nielson and D. Sands, Eds. Cham: Springer International Publishing, 2019, pp. 1-28.

[34] Q. Wang, A. Yahyavi, B. Kemme, and W. He, "I know what you did on your smartphone: Inferring app usage over encrypted data traffic," in 2015 IEEE Conference on Communications and Network Security (CNS), 2015, pp. 433-441.

[35] S. Zahur and D. Evans, "Obliv-c: A language for extensible data-oblivious computation," Cryptology ePrint Archive, Report 2015/1153, 2015, https://eprint.iacr.org/2015/1153.

[36] D. Zhang, A. Askarov, and A. C. Myers, "Languagebased control and mitigation of timing channels," in ACM SIGPLAN Conference on Programming Language Design and Implementation, PLDI '12, Beijing, China - June 11 - 16, 2012, 2012, pp. 99-110. [Online]. Available: https://doi.org/10.1145/2254064.2254078

[37] F. Zhang, W. He, X. Liu, and P. G. Bridges, "Inferring users' online activities through traffic analysis," in Proceedings of the Fourth ACM Conference on Wireless Network Security, ser. WiSec '11. New York, NY, USA: Association for Computing Machinery, 2011, p. 59-70. [Online]. Available: https://doi.org/10.1145/1998412.1998425 


\section{APPENDIX}

\section{A. Local configuration}

Lemma 6 (Progress-sensitive typing). Let $\Gamma$ be a typing environment, $p c, p c^{\prime}$ two levels and $c$ a command. If $\Gamma, p c \vdash c: p c^{\prime}$ then $p c \sqsubseteq p c^{\prime}$.

Proof: By induction on $c$ and monotinicity of $\sqsubseteq$.

Lemma 1 (Preservation of well-formedness). Let $\Gamma$ be a typing environment, $p c, p c^{\prime}$ be two levels, and $\langle c, m, I\rangle$ be a program configuration, such that the $\langle c, m, I\rangle$ is well-formed w.r.t. $\Gamma$, pc, and $p c^{\prime}$. Suppose this configuration takes a step

$$
\langle c, m, I\rangle \stackrel{t s}{\longrightarrow}_{\alpha}\left\langle c^{\prime}, m^{\prime}, I^{\prime}\right\rangle
$$

Then there exists $p c^{\prime \prime}$ such that $p c \sqsubseteq p c^{\prime \prime} \sqsubseteq p c^{\prime}$ and such that the resulting program configuration $\left\langle c^{\prime}, m^{\prime}, I^{\prime}\right\rangle$ is well-formed w.r.t. $\Gamma, p c^{\prime \prime}$, and $p c^{\prime}$.

Proof: The proof proceeds by induction on the structure of $c$.

Case skip: By SKIP we observe that $c^{\prime}$ is stop and $m=m^{\prime}$, hence we are done.

Case $c_{1} ; c_{2}$ : We case analyse on whether $c^{\prime}=c_{2}$. If $c^{\prime}=c_{2}$, we observe that $c^{\prime}$ was produced using SEQ-2 was taken and are done by the induction hypothesis, T-SEQ, Lemma 6 If $c^{\prime} \neq c_{2}$, we observe that $c^{\prime}$ was produced using SEQ-1 and are done by the induction hypothesis and T-SEQ.

Case $\mathrm{x}=\mathrm{e}$ : By Assign we have that $c^{\prime}$ is stop and $m^{\prime}=m[x \mapsto v]$ where $\langle e, m\rangle \Downarrow v$. Observing that T-Assign is the only applicable typing rule we have that $x \in \operatorname{dom}(\Gamma)$ and $t_{e}<: t_{x}$ by which we conclude that $m$ is well-formed w.r.t. $\Gamma$ and we are done.

Case $x=\operatorname{sizeof}(e)$ : By SIzEOF we observe $c^{\prime}$ is stop and $m^{\prime}=m[x \mapsto n]$ where $\langle e, m\rangle \Downarrow v$ and $n=\left\lceil\frac{\text { size }(v)}{\eta}\right\rceil$ and hence $n \in$ Int. By T-SIZEOF we have $\Gamma \vdash x:$ Int@ $\ell_{x}$, and hence $m$ is well-formed w.r.t. $\Gamma$ and we are done.

Case await $(r)$ : By AwAIT we observe that $c^{\prime}$ is stop and $m^{\prime}=m$, hence we are done.

Case $\operatorname{sleep}(e)$ : By SLEEP we observe that $c^{\prime}$ is await $(r)$ for some $r$ and $m^{\prime}=m$. As command await is trivially typed, we are done.

Case if $e$ then $c_{1}$ else $c_{2}$ : We observe that $m^{\prime}=m$ as neither IF-T nor IF-E alters the memory. We proceed by case analysis of $\langle e, m\rangle \Downarrow 0$, in both cases we are done by applying T-IF.

Case while $e$ do $c$ : We observe that WhILE transitions to a conditional branching with $m^{\prime}=m$. For the first branch, we observe that by T-WHILE we have $\Gamma \vdash e:$ Int@ $\ell$ and $\Gamma, p c \sqcup \ell \vdash c: p c^{\prime}$ hence we are done by T-IF using the induction hypothesis. For the other branch we are done by T-IF and T-SKIP using the induction hypothesis.

Case $x=\operatorname{in}(\ell)$ : By IN we have that $c^{\prime}$ is stop and $m(x) \in A$. We also have $m^{\prime}=m[x \mapsto v]$ for some $v \in A$ by definition of the choose function. Hence $m^{\prime}$ is well-type w.r.t. $\Gamma$ and we are done.

Case schedule $\left(\ell, e_{1}, e_{2}\right)$ : By Schedule we observe that $c^{\prime}$ is stop and $m^{\prime}=m$, hence we are done.

Case queue $(\ell, e)$ : By QUEUE we observe that $c^{\prime}$ is stop and $m^{\prime}=m$, hence we are done.

Lemma 7 (High program steps have only high effects). Given an attacker level $\ell_{a d v}$, a typing environment $\Gamma$, two levels pc, pc such that $p c \nsubseteq \ell_{a d v}$, and a program configuration $\langle c, m, I\rangle$ such that $\Gamma, p c \vdash c: p c^{\prime}$ and a timestamp ts such that

$$
\langle c, m, I\rangle \stackrel{t s}{\longrightarrow}_{\alpha}\left\langle c^{\prime}, m^{\prime}, I^{\prime}\right\rangle
$$

then $\lfloor\alpha\rfloor_{\ell_{a d v}}^{\bullet}=\epsilon$ and $m \approx_{\ell_{a d v}} m^{\prime}$ and $I \approx_{\ell_{a d v}}^{\bullet} I^{\prime}$.

Proof: We proceed by induction on command $c$.

Case skip: Trivially done by SKIP, observing that $m=m^{\prime}, I=I^{\prime}$ and $\alpha=\epsilon$.

Case $c_{1} ; c_{2}$ : We case on whether $c^{\prime}=c_{2}$. If false, we observe that the only matching rule in the operational semantics is SEQ-1 and are done by applying the induction hypothesis. If true, we observe that the only matching rule in the operational semantics is SEQ-2 and we are done by applying the induction hypothesis.

Case $\mathrm{x}=\mathrm{e}$ : We observe by ASSIGN that $I=I^{\prime}$ and have that $\alpha=\mathrm{a}(x, v)$ and $m^{\prime}=m[x \mapsto v]$. We must show $m \approx_{\ell_{a d v}} m^{\prime}$ and $\lfloor a(x, v)\rfloor_{\ell_{a d v}}^{\bullet}=\epsilon$.

By T-Assign we have (a) $\Gamma \vdash=\sigma_{e} @ \ell_{e}$, (b) $\Gamma(x)=\sigma_{x} @ \ell_{x}$, (c) $\sigma_{e} \nearrow p c<: \sigma_{t}$ and (d) $\ell_{e} \sqcup p c \sqsubseteq \ell_{x}$, hence in particular $\ell_{x} \nsubseteq \ell_{a d v}$. We case on $\sigma_{x}$ to pick the corresponding projection. If $\sigma_{x}=$ int we have $\lfloor\alpha\rfloor_{\ell_{a d v}}^{\bullet}=\epsilon$ and are done by observing that condition both conditions for memory equivalence is satisfied hence $m \approx_{\ell_{a d v}} m^{\prime}$. If $\sigma_{x}=\mathbf{s t r i n g} \ell^{\prime}$ we have by definition of $\nearrow$ and $<$ : that $\ell^{\prime} \nsubseteq \ell_{a d v}$ hence $\lfloor\alpha\rfloor_{\ell_{a d v}}^{\bullet}=\epsilon$ and we are done by observing $m \approx_{\ell_{a d v}} m^{\prime}$ by definition of memory equivalence. 
Case $x=\operatorname{sizeof}(e)$ : We observe by SizeOF that $I=I^{\prime}$ and $\alpha=\mathrm{a}(x, n)$ where $n=\left\lceil\frac{\operatorname{size}(v)}{\eta}\right\rceil$ for some $v$ such that $\langle e, m\rangle \Downarrow v$ and $m^{\prime}=m[x \mapsto v]$. We must show $m \approx_{\ell_{a d v}} m^{\prime}$ and $\lfloor\mathrm{a}(x, n)\rfloor_{\ell_{a d v}}^{\bullet}=\epsilon$.

By T-SizeOF we have (a) $\Gamma \vdash x:$ int $@ \ell_{x}$, (b) $\Gamma \vdash e: \sigma_{e} @ \ell_{e}^{a d v}$, (c) $p c \sqsubseteq \ell_{x}$, and (d) $\sigma_{e}=\operatorname{string}_{\ell^{\prime}} \Longrightarrow \ell^{\prime} \sqsubseteq \ell_{x}$. By (a) and the definition of memory equivalence we have $m \approx_{\ell_{a d v}} m^{\prime}$. By (c) and assumption $p c \nsubseteq \ell_{a d v}$ we have (e) $\ell_{x} \nsubseteq \ell_{a d v}$ hence by definition of internal event projection we have $[\mathrm{a}(x, n)\rfloor_{\ell_{\alpha d v}}^{\bullet}=\epsilon$ and we are done.

Case await $(r)$ : Trivially done by AWAIT, observing that $m=m^{\prime}, I=I^{\prime}$ and $\alpha=\epsilon$.

Case $\operatorname{sleep}(e)$ : Trivially done by SLEEP, observing that $m=m^{\prime}, I=I^{\prime}$ and $\alpha=\epsilon$.

Case if $e$ then $c_{1}$ else $c_{2}$ : We case on $\langle e, v\rangle \Downarrow 0$. If true then by IF-T, we observe that $m=m^{\prime}, I=I^{\prime}$, and $\alpha=\epsilon$ and we are done. Analogously by IF-E if false.

Case while $e$ do $c$ : Trivially done by WhILE, observing that $m=m^{\prime}, I=I^{\prime}$, and $\alpha=\epsilon$.

Case $x=\operatorname{in}(\ell)$ : We observe by IN that $I(\ell)=\vec{p}$, $\operatorname{choose}(\vec{p}, A, t s,[])=(v, \vec{q}), \alpha=\mathrm{i}(\ell, x, v), m^{\prime}=m[x \mapsto v]$, and $I^{\prime}=I[\ell \mapsto \vec{q}]$. We must show $\lfloor\mathrm{i}(\ell, x, v)\rfloor_{\ell_{a d v}}^{\bullet}=\epsilon, m \approx_{\ell_{a d v}} m^{\prime}$, and $I \approx_{\ell_{a d v}}^{\bullet} I^{\prime}$.

By T-IN we have (a) $\Gamma \vdash x: \sigma_{x} @ \ell_{x}$, (b) $p c \sqsubseteq \ell$, (c) $\sigma_{x} \nearrow \ell<: \sigma_{x}$, and (d) $\ell \sqsubseteq \ell_{x}$. By (b) and $p c \nsubseteq \ell_{a d v}$ we have (e) $\ell \nsubseteq \ell_{a d v}$ and hence by definition of internal event projection have $\lfloor\mathrm{i}(\ell, x, v)\rfloor_{\ell_{a d v}}=\epsilon$ and by definition of the choose and input environment equivalence have $I \approx_{\ell_{a d v}}^{\bullet} I^{\prime}$. To show memory equivalence, we observe that by (d) and we have $\ell_{x} \nsubseteq \ell_{a d v}$ and the first condition of memory equivalence is satisfied. To see that the second condition is satisfied we case on $\sigma_{x}=$ string $_{\ell^{\prime}}$. If false, we are done. If true, we have by (c) and the definition of $\nearrow$ and $<:$ that $\ell \sqsubseteq \ell^{\prime}$ and hence $\ell^{\prime} \nsubseteq \ell_{a d v}$ hence the second condition is satisfied and we have $m \approx_{\ell_{a d v}} m^{\prime}$ and we are done.

Case schedule $\left(\ell, e_{1}, e_{2}\right)$ : By T-SCHEDULE we have $p c=\perp$, leading to contradiction $\perp \nsubseteq \ell_{a d v}$, hence this case is impossible.

Case queue $(\ell, e)$ : We observe by Queve that $m=m^{\prime}, I=I^{\prime}$, and we have that $\alpha=\mathrm{q}(\ell, v)$ such that $\langle m, e\rangle \Downarrow v$. We must show $O \approx_{\ell_{a d v}} O^{\prime}$ and $\lfloor\mathrm{q}(\ell, v)\rfloor_{\ell_{a d v}}^{\bullet}=\epsilon$.

By T-QUEUE we have (b) $\Gamma \vdash e: \sigma_{e} @ \ell_{e}$ and (c) $\ell_{e} \sqcup \ell \sqsubseteq \ell$. By (c) and assumption $p c \nsubseteq \ell_{a d v}$ we have (d) $\ell \nsubseteq \ell_{a d v}$, hence by definition of internal event projection we have $\lfloor\mathrm{q}(\ell, v)\rfloor_{\ell_{a d v}}^{\bullet}=\epsilon$.

Lemma 8 (Noninterference for expressions). Given an attacker level $\ell_{\text {adv }}$, a typing environment $\Gamma$ and two memories $m_{1}$ and $m_{2}$ such that $m_{1} \approx_{\ell_{a d v}} m_{2}$, and an expression e such that $\Gamma \vdash e: \sigma @ \ell$, such that $\left\langle e, m_{1}\right\rangle \Downarrow v_{1}$ and $\left\langle e, m_{2}\right\rangle \Downarrow v_{2}$, then we have that

- $\ell \sqsubseteq \ell_{a d v} \Longrightarrow v_{1}=v_{2}$

- $\sigma=\operatorname{string}_{\ell^{\prime}} \wedge \ell^{\prime} \sqsubseteq \ell_{a d v} \Longrightarrow \operatorname{size}\left(v_{1}\right)=\operatorname{size}\left(v_{2}\right)$.

Proof: By induction on typing derivation $\Gamma \vdash e: \sigma @ \ell$ using the definition of $m_{1} \approx_{\ell_{a d v}} m_{2}$.

Lemma 4 (Program step noninterference). Given a level $\ell_{a d v}$ and a command $c$ and $\Gamma, p c, p c^{\prime}$ such that $c$ is well-formed w.r.t $\Gamma, p c, p c^{\prime}$, if

$$
\left\langle c, m_{1}, I_{1}\right\rangle \stackrel{t s}{\rightarrow}_{\alpha_{1}}\left\langle c^{\prime}, m_{1}^{\prime}, I_{1}^{\prime}\right\rangle
$$

then for any memory $m_{2}$ such that $m_{1} \approx_{\ell_{a d v}} m_{2}$ and input environment $I_{2}$ such that $I_{1} \approx_{\ell_{a d v}}^{\bullet} I_{2}$, we have that one of the following holds

1) either $\left\langle c, m_{2}, I_{2}\right\rangle \stackrel{t s}{\rightarrow}_{\alpha_{2}}\left\langle c^{\prime}, m_{2}^{\prime}, I_{2}^{\prime}\right\rangle$ such that $\left\lfloor\alpha_{1}\right\rfloor_{\ell_{a d v}}^{\bullet}=\left\lfloor\alpha_{2}\right\rfloor_{\ell_{a d v}}^{\bullet}$ and $m_{1}^{\prime} \approx_{\ell_{a d v}} m_{2}^{\prime}$ and $I_{1}^{\prime} \approx_{\ell_{a d v}}^{\bullet} I_{2}^{\prime}$.

2) or $\left\lfloor\alpha_{1}\right\rfloor_{\ell_{a d v}}^{\bullet}=\epsilon$ and there is $p c^{\prime \prime}$ such that $p c^{\prime \prime} \nsubseteq \ell_{a d v}$ and $\Gamma, p c^{\prime \prime} \vdash c: p c^{\prime}$ and $m_{1} \approx_{\ell_{a d v}} m_{1}^{\prime}$ and $I_{1} \approx_{\ell_{a d v}}^{\bullet} I_{1}^{\prime}$

Proof: The proof is by induction on the structure of command $c$.

Case skip: Trivially possible for both runs in with $c^{\prime}=$ stop and $\alpha_{1}=\alpha_{2}=\epsilon$. We are done by observing that no updates are made to memory or input environment.

Case $c_{1} ; c_{2}$ : There must be $c_{1}^{\prime}$ such that

$$
\left\langle c_{1}, m_{1}, I_{1}\right\rangle \stackrel{t s}{\rightarrow} \alpha_{1}\left\langle c_{1}^{\prime}, m_{1}^{\prime}, I_{1}^{\prime}\right\rangle
$$

and hence

$$
\left\langle c_{1} ; c_{2}, m_{1}, I_{1}\right\rangle \stackrel{t s}{\rightarrow}_{\alpha_{1}}\left\langle d, m_{1}^{\prime}, I_{1}^{\prime}\right\rangle
$$

where

$$
d= \begin{cases}c_{2} & \text { if } c_{1}^{\prime}=\text { stop } \\ c_{1}^{\prime} ; c_{2} & \text { otherwise }\end{cases}
$$


By T-SEQ we have $\Gamma, p c \vdash c_{1}: p c^{\prime \prime}$ and $\Gamma, p c^{\prime \prime} \vdash c_{2}: p c^{\prime}$. By induction hypothesis we have two cases for the second run. Either we have

$$
\left\langle c_{1} ; c_{2}, m_{2}, I_{2}\right\rangle \stackrel{t s}{\rightarrow}_{\alpha_{2}}\left\langle d, m_{2}^{\prime}, I_{2}^{\prime}\right\rangle
$$

such that $m_{1}^{\prime} \approx_{\ell_{a d v}} m_{2}^{\prime}$ and $I_{1}^{\prime} \approx_{\ell_{a d v}}^{\bullet} I_{2}^{\prime}$ and $\left\lfloor\alpha_{1}\right\rfloor_{\ell_{a d v}}^{\bullet}=\left\lfloor\alpha_{2}\right\rfloor_{\ell_{a d v}}^{\bullet}$ and we are done by the first condition. Or we have that $m_{1} \approx_{\ell_{a d v}} m_{1}^{\prime}$ and $I_{1} \approx_{\ell_{a d v}}^{\bullet} I_{1}^{\prime}$ and $\left\lfloor\alpha_{1}\right\rfloor_{\ell_{a d v}}^{\bullet}=\epsilon$, and existence of $p c^{\prime \prime \prime}$ such that $p c^{\prime \prime \prime} \nsubseteq \ell_{a d v}$ and $\Gamma ; p c^{\prime \prime \prime} \vdash c_{1}: p c^{\prime \prime}$. By this we have that $\Gamma ; p c^{\prime \prime \prime} \vdash c_{1} ; c_{2}: p c^{\prime}$ and we are done by the second condition.

Case $x=e$ : Trivially possible for both runs in one with $c^{\prime}=$ stop. We observe that no updates are made to input environment. By T-Assign we have $\Gamma \vdash x: \sigma_{x} @ \ell_{x}$ and $\Gamma \vdash e: \sigma_{e} @ \ell_{e}$ such that $\sigma_{e} \nearrow p c<: \sigma_{x}$ and $\ell_{e} \sqcup p c \sqsubseteq \ell_{x}$. As by AssigN we have $\alpha_{1}=\mathrm{a}\left(x, v_{1}\right)$ s.t. $\left\langle e, m_{1}\right\rangle \Downarrow v_{1}$ we consider the three cases for the internal event projection.

If $\left\lfloor\mathrm{a}\left(x, v_{1}\right)\right\rfloor_{\ell_{a d v}}^{\bullet}=\mathrm{a}\left(x, v_{1}\right)$ then by definition of internal event projection we have $\ell_{x} \sqsubseteq \ell_{a d v}$ and hence by Lemma 8 have $\left\langle e, m_{2}\right\rangle \Downarrow v_{2}$ s.t. $v_{1}=v_{2}$ and hence $\alpha_{2}=\alpha_{1}$. As $m_{1}^{\prime}=m_{1}\left[x \mapsto v_{1}\right]$ and $m_{2}^{\prime}=m_{2}\left[x \mapsto v_{2}\right]$ we are done by definition of memory equivalence.

If $\left\lfloor\mathrm{a}\left(x, v_{1}\right)\right\rfloor_{\ell_{\text {adv }}}^{\bullet}=|\mathrm{a}|\left(x, s_{1}\right)$ where $s_{1}=\operatorname{size}\left(v_{1}\right)$ then by definition of internal event projection we have $\sigma_{e}=$ string $_{\ell^{\prime}}$ such that $\ell^{\prime} \sqsubseteq \ell_{a d v}$ and hence by Lemma 8 have $\left\langle e, m_{2}\right\rangle \Downarrow v_{2}$ s.t. $\operatorname{size}\left(v_{1}\right)=\operatorname{size}\left(v_{2}\right)$ and hence $\left\lfloor\alpha_{2}\right\rfloor_{\ell_{a d v}}=\left\lfloor\alpha_{1}\right\rfloor_{\ell_{a d v}}^{\bullet}$. As $m_{1}^{\prime}=m_{1}\left[x \mapsto v_{1}\right]$ and $m_{2}^{\prime}=m_{2}\left[x \mapsto v_{2}\right]$ we are done by definition of memory equivalence.

If $\left\lfloor\mathrm{a}\left(x, v_{1}\right)\right\rfloor_{\ell_{q d v}}^{\bullet}=\epsilon$ then by definition of internal event projection we have $\ell_{x} \nsubseteq \ell_{a d v}$ and hence $\left\lfloor\alpha_{2}\right\rfloor_{\ell_{a d v}}^{\bullet}=\left\lfloor\alpha_{1}\right\rfloor_{\ell_{a d v}}^{\bullet}$. We are done by definition of memory equivalence.

Case $x=\operatorname{sizeof}(e)$ : Trivially possible for both runs in one with $c^{\prime}=$ stop. We observe that no updates are made to input environment.

By T-SizeOF we have $\Gamma \vdash x:$ int $@ \ell_{x}$ and $\Gamma \vdash e: \sigma_{e} @ \ell_{e}$ such that $\ell_{e} \sqcup p c \sqsubseteq \ell_{x}$. As by SIzEOF we have $\alpha_{1}=\mathrm{a}\left(x, n_{1}\right)$ s.t. $\left\langle e, m_{1}\right\rangle \Downarrow v_{1}$ and $n_{1}=\left\lceil\frac{\operatorname{size}\left(v_{1}\right)}{\eta}\right\rceil$ we consider the two cases for the internal event projection.

If $\left\lfloor\mathrm{a}\left(x, n_{1}\right)\right\rfloor_{\ell_{a d v}}^{\bullet}=\mathrm{a}\left(x, n_{1}\right)$ then by definition of the internal event projection we have $\ell_{x} \sqsubseteq \ell_{a d v}$. By T-SizEOF we have that if $\sigma_{e}=$ string $_{\ell^{\prime}}$ then $\ell^{\prime} \sqsubseteq \ell_{x}$ and by transitivity $\ell^{\prime} \sqsubseteq \ell_{a d v}$. By Lemma 8 we have $\left\langle e, m_{2}\right\rangle \Downarrow v_{2}$ such that $\operatorname{size}\left(v_{1}\right)=\operatorname{size}\left(v_{2}\right)$, and hence $\left\lfloor\alpha_{2}\right\rfloor_{\ell_{a d v}}^{\bullet}=\left\lfloor\alpha_{1}\right\rfloor_{\ell_{a d v}}^{\bullet}$. We are done by the definition of memory equivalence.

If $\left\lfloor\mathrm{a}\left(x, n_{1}\right)\right\rfloor_{\hat{\ell}_{a d v}}^{\bullet}=\epsilon$ then by definition of internal event projection we have $\ell_{x} \nsubseteq \ell_{a d v}$ and hence $\left\lfloor\alpha_{2}\right\rfloor_{\ell_{a d v}}^{\bullet}=\left\lfloor\alpha_{1}\right\rfloor_{\ell_{a d v}}^{\bullet}$. We are done by definition of memory equivalence.

Case await $(r)$ : It must be that $r \leq t s$ hence this transition is possible in both runs with $c^{\prime}=$ stop. As no event is emitted, we are done.

Case $\operatorname{sleep}(e)$ : If $p c^{\prime} \sqsubseteq \ell_{a d v}$ then by SLEEP we have that if $\left\langle e, m_{1}\right\rangle \Downarrow w$ then

$$
\left\langle\operatorname{sleep}(e), m_{1}, I_{1}\right\rangle \stackrel{t s}{\rightarrow}{ }_{\epsilon}\left\langle\operatorname{await}(t s+w), m_{1}, I_{1}\right\rangle
$$

By T-SLEEP we have $\Gamma \vdash e:$ int $@ \ell$ and $p c^{\prime}=p c \sqcup \ell$ and by assumption $p c^{\prime} \sqsubseteq \ell_{a d v}$ we therefore have $\ell \sqsubseteq \ell_{a d v}$. By Lemma 8 this gives us $\left\langle e, m_{2}\right\rangle \Downarrow w$ and hence

$$
\left\langle\operatorname{sleep}(e), m_{2}, I_{2}\right\rangle \stackrel{t s}{\rightarrow}{ }_{\epsilon}\left\langle\operatorname{await}(t s+w), m_{2}, I_{2}\right\rangle
$$

If $p c^{\prime} \nsubseteq \ell_{a d v}$, we observe that by T-SLEEP we have $\Gamma, p c^{\prime} \vdash \operatorname{sleep}(e): p c^{\prime}$ and we are done observing that by SLEEP, $\alpha_{1}=\epsilon$, and memory and input are unchanged.

Case if $e$ then $c_{1}$ else $c_{2}$ : By T-IF we have $\Gamma \vdash e:$ int@ $\ell$ and $\Gamma ; p c \sqcup \ell \vdash c_{1}: p c_{1}$ and $\Gamma ; p c \sqcup \ell \vdash c_{2}: p c_{2}$ such that $p c^{\prime}=p c_{1} \sqcup p c_{2}$. If $\ell \sqcup \ell_{a d v}$, then by Lemma 8 we have some $v$ such that $\left\langle e, m_{1}\right\rangle \Downarrow v$ and $\left\langle e, m_{2}\right\rangle \Downarrow v$, hence both runs transition to the same command and we are done by observing $\alpha_{1}=\alpha_{2}=\epsilon$ and memory and input environments are unchanged. If $\ell \nsubseteq \ell_{a d v}$ we observe again by T-IF that $\Gamma, p c \sqcup \ell \vdash$ if $e$ then $c_{1}$ else $c_{2}: p c^{\prime}$ and we are done.

Case while $e$ do $c$ : Trivial.

Case $x=$ in $(\ell)$ : By T-IN we observe that $p c^{\prime}=\ell$. If $\ell \sqsubseteq \ell_{a d v}$, then by definition of input environment equivalence and function choose we have that both runs obtain same input value at the same time, therefore producing same events and making same updates to the input environment. We are done by definitions of memory equivalence and input environment equivalence.

If $\ell \nsubseteq \ell_{a d v}$, we observe that by T-IN we have $\Gamma, \ell \vdash x=$ in $(\ell): \ell$. By IN we have some $v, \vec{q}$ such that $\alpha_{1}=\mathrm{i}(\ell, x, v)$, $m_{1}^{\prime}=m_{1}[x \mapsto v]$ and $I_{1}^{\prime}=I_{1}[\ell \mapsto \vec{q}]$ and by definition of the choose function have $I_{1} \approx_{\ell_{a d v}}^{\bullet} I_{1}^{\prime}$. By definition internal event projection we have $\left\lfloor\alpha_{1}\right\rfloor_{\ell_{a d v}}^{\bullet}=\epsilon$ and we are done by definition of memory, satisfying the second condition.

Case schedule $\left(\ell, e_{1}, e_{2}\right)$ : Trivially possible in both runs updating only schedules. By T-SCHEDULE we have $\Gamma \vdash e_{1}:$ int @ $\perp$ and $\Gamma \vdash e_{2}$ : int @ hence by Lemma 8 we have $a, w$ such that $\left\langle e_{1}, m_{1}\right\rangle \Downarrow a$ and $\left\langle e_{1}, m_{2}\right\rangle \Downarrow a$, and $\left\langle e_{2}, m_{1}\right\rangle \Downarrow w$ and $\left\langle e_{2}, m_{2}\right\rangle \Downarrow w$. As $\alpha_{1}=\mathrm{s}(\ell, a, w)=\alpha_{2}$ we are done. 
Case queue $(\ell, e)$ : Trivially possible in both runs updating only output environments. By QuEUE we have $\left\langle e, m_{1}\right\rangle \Downarrow v_{1}$ and $\alpha_{1}=\mathrm{q}\left(\ell, v_{1}\right)$. We consider the two cases of the internal event projection.

If $\left\lfloor\mathrm{q}\left(\ell, v_{1}\right)\right\rfloor_{\ell_{a d v}}^{\bullet}=\mathrm{q}\left(\ell, v_{1}\right)$ then by definition of the internal event projection we have $\ell \sqsubseteq \ell_{a d v}$. By T-QUEUE we have $\Gamma \vdash e: \sigma_{e} @ \ell_{e}$ and $\ell_{e} \sqcup p c \sqsubseteq \ell$ hence in particular $\ell_{e} \sqsubseteq \ell_{a d v}$ and therefore by Lemma 8 we have $\left\langle e, m_{2}\right\rangle \Downarrow v_{2}$ such that $v_{1}=v_{2}$ by which we have $\alpha_{2}=\alpha_{1}$ and we are done.

If $\left\lfloor q\left(\ell, v_{1}\right)\right\rfloor_{\ell_{a d v}}^{\bullet}=\epsilon$ then by definition of internal event projection we have $\ell \nsubseteq \ell_{a d v}$, and therefore also $\left\lfloor\alpha_{2}\right\rfloor_{\ell_{a d v}}=\epsilon$ and we are done.

\section{B. Global configuration}

Lemma 9 (High update preserves equivalence). For any level $\ell, \ell_{a d v}$, program event $\alpha$, output environments $O, O^{\prime}$, and schedule $\pi$, if $\lfloor\alpha\rfloor_{\ell_{a d v}}^{\bullet}=\epsilon$ and upd $(O, \pi, \alpha)=\left(O^{\prime}, \pi^{\prime}\right)$ then $O \approx_{\ell_{a d v}} O^{\prime}$ and $\pi^{\prime}=\pi$.

Proof: By definition of upd, internal event projection and output environment equivalence.

Lemma 10 (Sending preserves output environment equivalence). For any levels $\ell, \ell_{a d v}$, schedule $\pi$, timestamp ts, and output environments $O_{1}, O_{2}$ such that $O_{1} \approx_{\ell_{a d v}} O_{2}$, if $\pi(t s)=\ell, \operatorname{send}\left(O_{1}, \ell\right)=\left(\beta_{1}, O_{1}^{\prime}\right)$ and $\operatorname{send}\left(O_{2}, \ell\right)=\left(\beta_{2}, O_{2}^{\prime}\right)$ then $O_{1}^{\prime} \approx_{\ell_{a d v}} O_{2}^{\prime}$ and $\left\lfloor\beta_{1}\right\rfloor_{\ell_{a d v}}=\left\lfloor\beta_{2}\right\rfloor_{\ell_{a d v}}$.

Proof: If $\ell \sqsubseteq \ell_{a d v}$ then by definition of output environment equivalence we have $O_{1}(\ell)=O_{2}(\ell)$ hence send $\left(O_{1}, \ell\right)=$ send $\left(\mathrm{O}_{2}, \ell\right)$ which concludes the case.

If (a) $\ell \nsubseteq \ell_{a d v}$, then there is $p_{1}, \vec{q}_{1}$ such that send $\left(O_{1}, \ell\right)=\left(\mathrm{o}\left(\ell, p_{1}\right), \vec{q}_{1}\right)$ and $p_{2}, \vec{q}_{2}$ such that $\operatorname{send}\left(O_{2}, \ell\right)=\left(\mathrm{o}\left(\ell, p_{2}\right), \vec{q}_{2}\right)$. By (a) and by definition of event projection, we have $\left\lfloor\mathrm{o}\left(\ell, p_{1}\right)\right\rfloor_{\ell_{a d v}}=\mathrm{o}(\ell,-)=\left\lfloor\mathrm{o}\left(\ell, p_{2}\right)\right\rfloor_{\ell_{\text {adv }}}$ and by (a) and definition of output environment equivalence we have $O_{1}\left[\ell \mapsto \vec{q}_{1}\right] \approx_{\ell_{a d v}} O_{2}\left[\ell \mapsto \vec{q}_{2}\right]$ which concludes the case.

Lemma 5 (Global step noninterference). Given a level $\ell_{a d v}$ and a command $c$ and $\Gamma, p c, p c^{\prime}$ such that $c$ is well-formed w.r.t $\Gamma, p c, p c^{\prime}$, if

$$
\left.\backslash\left\langle c, m_{1}, I_{1}\right\rangle, O_{1}, \pi, t s \downarrow \rightarrow_{\left(t s: \alpha_{1}, \beta_{1}\right)} \backslash\left\langle c^{\prime}, m_{1}^{\prime}, I_{1}^{\prime}\right\rangle, O_{1}^{\prime}, \pi^{\prime}, t s^{\prime}\right\rangle
$$

then for any memory $m_{2}$ such that $m_{1} \approx_{\ell_{a d v}} m_{2}$; input environment $I_{2}$ such that $I_{1} \approx_{\ell_{a d v}}^{\bullet} I_{2}$; and output environment $O_{2}$ such that $\mathrm{O}_{1} \approx_{\ell_{a d v}} \mathrm{O}_{2}$, we have that one of the following holds

1) either

$$
\left.\left.\backslash\left\langle c, m_{2}, I_{2}\right\rangle, O_{2}, \pi, t s\right\rangle \rightarrow_{\left(t s: \alpha_{2}, \beta_{2}\right)} \backslash\left\langle c^{\prime}, m_{2}^{\prime}, I_{2}^{\prime}\right\rangle, O_{2}^{\prime}, \pi^{\prime}, t s^{\prime}\right\rangle
$$

such that each of the following hold
a) $\left\lfloor\alpha_{1}\right\rfloor_{\ell_{a d v}}^{\bullet}=\left\lfloor\alpha_{2}\right\rfloor_{\ell_{a d v}}^{\bullet}$
b) $\left\lfloor\beta_{1}\right\rfloor \ell_{a d v}=\left\lfloor\beta_{2}\right\rfloor \ell_{a d v}$
c) $m_{1}^{\prime} \approx_{\ell_{a d v}} m_{2}^{\prime}$
d) $I_{1}^{\prime} \approx_{\ell_{a d v}}^{\bullet a d v} I_{2}^{\prime}$
e) $O_{1}^{\prime} \approx_{\ell_{a d v}} O_{2}^{\prime}$

2) or there is $p c^{\prime \prime}$ such that $p c^{\prime \prime} \nsubseteq \ell_{a d v}$ and c is well-formed w.r.t $\Gamma, p c^{\prime \prime}, p c^{\prime}$, and such that each of the following hold

a) $\left\lfloor\alpha_{1}\right\rfloor_{\ell_{a d v}}^{\bullet}=\epsilon$

b) $m_{1} \approx_{\ell_{a d v}} m_{1}^{\prime}$

c) $I_{1} \approx_{\ell_{a d v}}^{\bullet a d v} I_{1}^{\prime}$

d) $\pi=\pi^{\prime}$

e) $\beta_{1} \neq \epsilon$ if and only if there are $\beta_{2}$ and $O_{2}^{\prime}$ such that $\left\lfloor\beta_{1}\right\rfloor_{\ell_{a d v}}=\left\lfloor\beta_{2}\right\rfloor \ell_{a d v}, O_{1}^{\prime} \approx_{\ell_{a d v}} O_{2}^{\prime}$, and

$$
\begin{aligned}
& \backslash\left\langle\text { stop, } m_{2}, I_{2}\right\rangle, O_{2}, \pi, t s \downarrow \\
& \quad \rightarrow\left(t s: \epsilon, \beta_{2}\right) \\
& \quad\left\langle\left\langle\text { stop }, m_{2}, I_{2}\right\rangle, O_{2}^{\prime}, \pi, t s^{\prime}\right\rangle
\end{aligned}
$$

Proof: We case on the global step for the first run.

Case G-STEP: By Lemma 4 we have

(1) either $\left\langle c, m_{2}, I_{2}\right\rangle \stackrel{t s}{\rightarrow} \alpha_{2}\left\langle c^{\prime}, m_{2}^{\prime}, I_{2}^{\prime}\right\rangle$ such that $\left\lfloor\alpha_{1}\right\rfloor_{\ell_{a d v}}^{\bullet}=\left\lfloor\alpha_{2}\right\rfloor_{\ell_{a d v}}^{\bullet}$ and $m_{1}^{\prime} \approx_{\ell_{a d v}} m_{2}^{\prime}$ and $I_{1}^{\prime} \approx_{\ell_{a d v}}^{\bullet} I_{2}^{\prime}$

(2) or $\left\lfloor\alpha_{1}\right\rfloor_{\ell_{a d v}}^{\bullet}=\epsilon$ and there is $p c^{\prime \prime}$ such that $p c^{\prime \prime} \nsubseteq \ell_{a d v}$ and $\Gamma ; p c^{\prime \prime} \vdash c: p c^{\prime}$ and $m_{1} \approx_{\ell_{a d v}} m_{1}^{\prime}$ and $I_{1} \approx_{\ell_{a d v}}^{\bullet} I_{1}^{\prime}$

If (1), we inspect $\left\lfloor\alpha_{1}\right\rfloor_{\ell_{a d v}}^{\bullet}$. If $\left\lfloor\alpha_{1}\right\rfloor_{\ell_{a d v}}^{\bullet}=\epsilon$ then we apply Lemma 9 in both runs to get $O_{1}^{\prime} \approx_{\ell_{a d v}} O_{1} \approx_{\ell_{a d v}} O_{2} \approx_{\ell_{a d v}} O_{2}^{\prime}$. We are done by Lemma 10 satisfying condition 1. If $\left\lfloor\alpha_{1}\right\rfloor_{\ell_{a d v}}^{\bullet} \neq \epsilon$ we are done by definition of upd, observing that the same update is made in each run, and by Lemma 10. 
If (2), we inspect $t s \in \operatorname{dom}(\pi)$.

If $t s \notin \operatorname{dom}(\pi)$ then as $\left\lfloor\alpha_{1}\right\rfloor_{\ell_{a d v}}^{\cdot}=\epsilon$ we get by Lemma $\bigvee$ that $O_{1} \approx_{\ell_{a d v}} O_{1}^{\prime}$. Further, we have by definition of internal event projection and definition of upd that $\pi^{\prime}=\pi$, hence $t s \notin \operatorname{dom}\left(\pi^{\prime}\right)$ and by definition of send we have $\beta_{1}=\epsilon$ and we are done satisfying condition 2 .

If $\pi(t s)=\ell$ we show that we satisfy condition 2 and consider each direction

$(\rightarrow)$ As the first run steps by G-STEP we have by Lemma 9 that upd $\left(O_{1}, \pi, \alpha\right)=\left(O_{1}^{\prime \prime}, \pi\right)$ such that $O_{1} \approx_{\ell_{a d v}} O_{1}^{\prime \prime}$, and by Lemma 10 have $\operatorname{send}\left(O_{1}^{\prime \prime}, \ell\right)=\left(\beta_{1}, O_{1}^{\prime}\right)$ and send $\left(O_{2}, \ell\right)=\left(\beta_{2}, O_{2}^{\prime}\right)$ such that $O_{1}^{\prime} \approx_{\ell_{a d v}} O_{2}^{\prime}$ and $\left\lfloor\beta_{1}\right\rfloor_{\ell_{a d v}}=\left\lfloor\beta_{2}\right\rfloor_{\ell_{a d v}}$ and hence

$$
\left.\left.\backslash\left\langle\text { stop, } m_{2}, I_{2}\right\rangle, O_{2}, \pi, t s\right\rangle \rightarrow_{\left(t s: \epsilon, \beta_{2}\right)} \backslash\left\langle\text { stop, } m_{2}, I_{2}\right\rangle, O_{2}^{\prime}, \pi, t s^{\prime}\right\rangle
$$

and we are done.

$(\leftarrow)$ As $\beta_{2} \neq \epsilon$, the anchor-configuration steps G-STOP and we apply Lemma 9 in the first run and Lemma 10 in both runs to get $\left\lfloor\beta_{1}\right\rfloor_{\ell_{a d v}}=\left\lfloor\beta_{2}\right\rfloor_{\ell_{a d v}}$ and $O_{1}^{\prime} \approx_{\ell_{a d v}} O_{2}^{\prime}$ and we are done.

Case G-BLOCK: We have $\alpha_{1}=\epsilon$ and $m_{1}=m_{1}^{\prime}$ and $I_{1}=I_{1}^{\prime}$ and $O_{1}=O_{1}^{\prime}$ and $\pi=\pi^{\prime}$. We consider the possible steps of the second run.

Case G-STEP: We will show that condition 2 is satisfied. To this end, we first apply the induction hypothesis in the second run to get $p c^{\prime \prime}$ such that $p c^{\prime \prime} \nsubseteq \ell_{a d v}$ and $\Gamma ; p c^{\prime \prime} \vdash c: p c^{\prime}$. We proceed by inspecting $t s \in \operatorname{dom}(\pi)$.

If $t s \notin \operatorname{dom}(\pi)$ then by definition of send we have $\beta_{1}=\epsilon$ and we are done.

If $\pi(t s)=\ell$ we consider each direction

$(\rightarrow)$ We have by Lemma 10 have send $\left(O_{1}, \ell\right)=\left(\beta_{1}, O_{1}^{\prime}\right)$ and send $\left(O_{2}, \ell\right)=\left(\beta_{2}, O_{2}^{\prime}\right)$ such that $O_{1}^{\prime} \approx_{\ell_{a d v}} O_{2}^{\prime}$ and $\left\lfloor\beta_{1}\right\rfloor_{\ell_{a d v}}=\left\lfloor\beta_{2}\right\rfloor_{\ell_{a d v}}$ and hence

$$
\left.\backslash\left\langle\text { stop, } m_{2}, I_{2}\right\rangle, O_{2}, \pi, t s \downarrow \rightarrow_{\left(t s: \epsilon, \beta_{2}\right)} \backslash\left\langle\text { stop, } m_{2}, I_{2}\right\rangle, O_{2}^{\prime}, \pi, t s^{\prime}\right\rangle
$$

and we are done.

$(\leftarrow)$ As $\beta_{2} \neq \epsilon$, the anchor-configuration steps G-STOP and we apply Lemma 10 in both runs to get $\left\lfloor\beta_{1}\right\rfloor_{\ell_{a d v}}=\left\lfloor\beta_{2}\right\rfloor_{\ell_{a d v}}$ and $O_{1}^{\prime} \approx_{\ell_{a d v}} O_{2}^{\prime}$ and we are done.

Case G-BLOCK: Immediate by Lemma 10

Case G-STOP: Immediate by Lemma 10

Case No step: It must be that $t s \notin \operatorname{dom}(\pi)$ and hence by definition of send have $\beta_{1}=\epsilon$ and we are done.

Case G-Stop: We have $\alpha_{1}=\epsilon$ and $m_{1}=m_{1}^{\prime}$ and $I_{1}=I_{1}^{\prime}$ and $O_{1}=O_{1}^{\prime}$ and $\pi=\pi^{\prime}$. We consider the possible steps of the second run.

Case G-STEP: We will show that condition 2 is satisfied. To this end, we first apply the induction hypothesis in the second run to get $p c^{\prime \prime}$ such that $p c^{\prime \prime} \nsubseteq \ell_{a d v}$ and $\Gamma ; p c^{\prime \prime} \vdash c: p c^{\prime}$. We proceed by inspecting $t s \in \operatorname{dom}(\pi)$.

If $t s \notin \operatorname{dom}(\pi)$ then by definition of send we have $\beta_{1}=\epsilon$ and we are done.

If $\pi(t s)=\ell$ we consider each direction

$(\rightarrow)$ We have by Lemma 10 have send $\left(O_{1}, \ell\right)=\left(\beta_{1}, O_{1}^{\prime}\right)$ and $\operatorname{send}\left(O_{2}, \ell\right)=\left(\beta_{2}, O_{2}^{\prime}\right)$ such that $O_{1}^{\prime} \approx_{\ell_{\text {adv }}} O_{2}^{\prime}$ and $\left\lfloor\beta_{1}\right\rfloor_{\ell_{a d v}}=\left\lfloor\beta_{2}\right\rfloor_{\ell_{a d v}}$ and hence

$$
\left.\left.\backslash\left\langle\text { stop, } m_{2}, I_{2}\right\rangle, O_{2}, \pi, t s\right\rangle \rightarrow{ }_{\left(t s: \epsilon, \beta_{2}\right)} \backslash\left\langle\text { stop, } m_{2}, I_{2}\right\rangle, O_{2}^{\prime}, \pi, t s^{\prime}\right\rangle
$$

and we are done.

$(\leftarrow)$ As $\beta_{2} \neq \epsilon$, the anchor-configuration steps G-STOP and we apply Lemma 10 in both runs to get $\left\lfloor\beta_{1}\right\rfloor_{\ell_{a d v}}=\left\lfloor\beta_{2}\right\rfloor_{\ell_{a d v}}$ and $O_{1}^{\prime} \approx_{\ell_{a d v}} O_{2}^{\prime}$ and we are done.

Case G-BLOCK: Immediate by Lemma 10

Case G-STOP: Immediate by Lemma 10

Case No step: It must be that $t s \notin \operatorname{dom}(\pi)$ and hence by definition of send have $\beta_{1}=\epsilon$ and we are done.

\section{Soundness of security type system}

Lemma 2 (Internal knowledge refines external knowledge). For any program configuration $P$, trace $\tau$, and level $\ell_{\text {adv }}$, the knowledge of an external attacker is less precise than the knowledge of an internal attacker. That is,

$$
k\left(P, \tau, \ell_{a d v}\right) \supseteq k^{\bullet}\left(P, \tau, \ell_{a d v}\right)
$$
show

Proof: Given an $P_{2}$ s.t. $P \approx_{\ell_{a d v}} P_{2}$ and unfolding the definitions of attacker knowledge and internal knowledge we must

$$
\left(\tau \vdash^{\bullet} \ell_{a d v}\right)=\left(\tau_{2} \vdash^{\bullet} \ell_{a d v}\right) \Longrightarrow\left(\tau \uparrow \ell_{a d v}\right)=\left(\tau_{2}\left\lceil\ell_{a d v}\right)\right.
$$


The proof is by induction on trace $\tau$.

Base case $\tau=\epsilon$ : Immediate.

Inductive case $\tau=\tau^{\prime} \cdot(t s: \alpha, \beta)$ : We have $\tau_{2}=\tau_{2}^{\prime} \cdot\left(t s_{2}: \alpha_{2}, \beta_{2}\right)$ and have IH

$$
\left(\tau^{\prime} \Gamma^{\bullet} \ell_{a d v}\right)=\left(\tau_{2}^{\prime} \Gamma^{\bullet} \ell_{a d v}\right) \Longrightarrow\left(\tau^{\prime} \mid \ell_{a d v}\right)=\left(\tau_{2}^{\prime} \mid \ell_{a d v}\right)
$$

By assumption, we have $\left(\tau^{\prime} \cdot(t s: \alpha, \beta) \uparrow^{\bullet} \ell_{a d v}\right)=\left(\tau_{2}^{\prime} \cdot\left(t s_{2}: \alpha_{2}, \beta_{2}\right) \uparrow^{\bullet} \ell_{a d v}\right)$ and hence $\lfloor\alpha\rfloor_{\ell_{a d v}}^{\bullet}=\left\lfloor\alpha_{2}\right\rfloor_{\ell_{a d v}}^{\bullet}$ and $\lfloor\beta\rfloor_{\ell_{a d v}}=\left\lfloor\beta_{2}\right\rfloor_{\ell_{a d v}}$ and we consider the two results of the internal trace filtering:

Case $\left(\tau \vdash^{\bullet} \ell_{a d v}\right)=\left(\tau^{\prime} \Gamma^{\bullet} \ell_{a d v}\right)$ : By definition of internal trace filtering have $\lfloor\alpha\rfloor_{\ell_{a d v}}^{\bullet}=\lfloor\beta\rfloor_{\ell_{a d v}}^{\bullet}=\epsilon$, and hence definition of trace filtering we have $\left(\tau \mid \ell_{a d v}\right)=\left(\tau^{\prime} \mid \ell_{a d v}\right)$ and $\left(\tau_{2}\left\lceil\ell_{a d v}\right)=\left(\tau_{2}^{\prime}\left\lceil\ell_{a d v}\right)\right.\right.$ and we are done by IH.

Case $\left(\tau r^{\bullet} \ell_{a d v}\right) \neq\left(\tau^{\prime} \vdash^{\bullet} \ell_{a d v}\right)$ : We have $t s=t s_{2}$ and hence $\left(t s:\lfloor\alpha\rfloor_{\ell_{a d v}}^{\bullet}\lfloor\beta\rfloor_{\ell_{a d v}}\right)=\left(t s_{2}:\left\lfloor\alpha_{2}\right\rfloor_{\ell_{a d v}}\left\lfloor\beta_{2}\right\rfloor_{\ell_{a d v}}\right)$. From this, we trivially get $\left(t s: \epsilon,\lfloor\beta\rfloor_{\ell_{a d v}}\right)=\left(t s_{2}: \epsilon,\left\lfloor\beta_{2}\right\rfloor_{\ell_{a d v}}\right)$ and are done by definition of trace filtering and IH.

Theorem 1 (Soundness). Given a typing environment $\Gamma$, two levels $p c, p c^{\prime}$, and a program configuration $P$ that is well-formed w.r.t $\Gamma, p c, p c^{\prime}$, the run $\backslash P, O_{\text {init }}, \pi_{\text {init }}, 0 \triangleright \rightarrow_{\tau}^{*}\left\langle P^{\prime}, O^{\prime}, \pi^{\prime}, t s^{\prime} \backslash\right.$ satisfies Definition 8

Proof: By Definition 8 and unfolding of program configuration $P$ we are given a program $c$, initial memory $m$ and initial input environment $I$ such that

$$
\left.\left.\backslash\langle c, m, I\rangle, O_{\text {init }}, \pi_{\text {init }}, 0\right\rangle \rightarrow_{\tau \cdot \gamma}^{*} \backslash\left\langle c^{\prime}, m^{\prime}, I^{\prime}\right\rangle, O^{\prime}, \pi^{\prime}, t s^{\prime}\right\rangle
$$

and must show that for any $\ell_{a d v}$ we have

$$
k\left(c, m, I, \tau \cdot \gamma, \ell_{a d v}\right) \supseteq k\left(c, m, I, \tau, \ell_{a d v}\right)
$$

Let $\left(m_{2}, I_{2}\right) \in k\left(c, m, I, \tau, \ell_{a d v}\right)$ and $\ell_{a d v}$ be given. Unfolding the definition of attacker knowledge we have $\tau_{2}$ such that $\left(\tau\left\lceil\ell_{a d v}\right)=\left(\tau_{2}\left\lceil\ell_{a d v}\right)\right.\right.$ and

$$
\left.\left.\backslash\left\langle c, m_{2}, I_{2}\right\rangle, O_{\text {init }}, \pi_{\text {init }}, 0\right\rangle \rightarrow_{\tau_{2}}^{*} \backslash\left\langle c_{2}^{\prime}, m_{2}^{\prime}, I_{2}^{\prime}\right\rangle, O_{2}^{\prime}, \pi_{2}^{\prime}, t s_{2}^{\prime}\right\rangle
$$

We must show that

$$
\left.\left.\checkmark\left\langle c, m_{2}, I_{2}\right\rangle, O_{\text {init }}, \pi_{\text {init }}, 0\right\rangle \rightarrow_{\tau_{2} \cdot \gamma_{2}}^{*} \backslash\left\langle c_{2}^{\prime}, m_{2}^{\prime}, I_{2}^{\prime}\right\rangle, O_{2}^{\prime}, \pi_{2}^{\prime}, t s_{2}^{\prime}\right\rangle
$$

such that $\left(\tau \cdot \gamma \uparrow \ell_{a d v}\right)=\left(\tau_{2} \cdot \gamma_{2} \uparrow \ell_{a d v}\right)$.

We do this by showing a stronger property using internal trace filtering and applying Lemma 2. We show that if

$$
\begin{aligned}
\left.\backslash\left\langle c, m_{1}, I_{1}\right\rangle, O_{\text {init }}, \pi_{\text {init }}, 0\right\rangle & \rightarrow_{\tau}^{n} \backslash\left\langle c_{1}^{\prime}, m_{1}^{\prime}, I_{1}^{\prime}\right\rangle, O_{1}^{\prime}, \pi^{\prime}, n \searrow \\
& \left.\rightarrow{ }_{\gamma} \backslash\left\langle c_{1}^{\prime \prime}, m_{1}^{\prime \prime}, I_{1}^{\prime \prime}\right\rangle, O_{1}^{\prime \prime}, \pi^{\prime \prime}, n+1\right\rangle
\end{aligned}
$$

then one of the following holds

(1) either

$$
\begin{aligned}
\left.\backslash\left\langle c, m_{2}, I_{2}\right\rangle, O_{\text {init }}, \pi_{\text {init }}, 0\right\rangle & \left.\rightarrow{ }_{\tau_{2}}^{n} \backslash\left\langle c_{2}^{\prime}, m_{2}^{\prime}, I_{2}^{\prime}\right\rangle, O_{2}^{\prime}, \pi^{\prime}, n\right\rangle \\
& \left.\rightarrow \gamma_{2} \backslash\left\langle c_{2}^{\prime \prime}, m_{2}^{\prime \prime}, I_{2}^{\prime \prime}\right\rangle, O_{2}^{\prime \prime}, \pi^{\prime \prime}, n+1\right\rangle
\end{aligned}
$$

s.t. $\left(\tau \cdot \gamma \Gamma^{\bullet} \ell_{a d v}\right)=\left(\tau_{2} \cdot \gamma_{2} \Gamma^{\bullet} \ell_{a d v}\right)$ and $m_{1}^{\prime \prime} \approx_{\ell_{a d v}} m_{2}^{\prime \prime}$ and $I_{1}^{\prime \prime} \approx_{\ell_{a d v}}^{\bullet} I_{2}^{\prime \prime}$ and $O_{1}^{\prime \prime} \approx_{\ell_{a d v}} O_{2}^{\prime \prime}$

(2) or there is $n^{\prime} \leq n$

$$
\left.\varangle\left\langle c, m_{2}, I_{2}\right\rangle, O_{\text {init }}, \pi_{\text {init }}, 0 \triangleright \rightarrow{ }_{\tau_{2}}^{n^{\prime}} \triangleleft\left\langle c_{2}^{\prime}, m_{2}^{\prime}, I_{2}^{\prime}\right\rangle, O_{2}^{\prime}, \pi^{\prime}, n^{\prime}\right\rangle
$$

s.t. $\left(\tau \cdot \gamma \vdash^{\bullet} \ell_{a d v}\right)=\left(\tau r^{\bullet} \ell_{a d v}\right)=\left(\tau_{2} \Gamma^{\bullet} \ell_{a d v}\right)$ and $m_{1}^{\prime \prime} \approx_{\ell_{a d v}} m_{2}^{\prime}$ and $I_{1}^{\prime \prime} \approx_{\ell_{a d v}}^{\bullet} I_{2}^{\prime}$ and $O_{1}^{\prime \prime} \approx_{\ell_{a d v}} O_{2}^{\prime}$ and $\nexists t s^{\prime} \in \operatorname{dom}\left(\pi^{\prime}\right)$ : $t s^{\prime} \geq n$ and there is $p c_{1}, p c_{2}$ such that $p c_{1} \nsubseteq \ell_{a d v}$ and $p c_{2} \nsubseteq \ell_{a d v}$ and $c_{1}^{\prime}$ is well-formed w.r.t $\Gamma, p c_{1}, p c^{\prime}$ and and $c_{2}^{\prime}$ is well-formed w.r.t $\Gamma, p c_{2}, p c^{\prime}$

In each case, the equality of traces using internal trace filtering and Lemma 2 gives us equality of traces using trace filtering as desired.

The proof is by induction on $n$

Base case $n=0$ : Immediate by Definition 7, Definition 13, Lemma 3, and Lemma 5 ,

Inductive case $n>0$ : We apply the induction hypothesis and get

(1) either

$$
\left.\triangleleft\left\langle c, m_{2}, I_{2}\right\rangle, O_{i n i t}, \pi_{i n i t}, 0 \triangleright \rightarrow \tau_{2}^{n-1} \triangleleft\left\langle c_{2}^{\prime}, m_{2}^{\prime}, I_{2}^{\prime}\right\rangle, O_{2}^{\prime}, \pi^{\prime}, n-1\right\rangle
$$


s.t. $\left(\tau\left\lceil\ell_{a d v}\right)=\left(\tau_{2} \uparrow \ell_{a d v}\right)\right.$ and $m_{1}^{\prime} \approx_{\ell_{a d v}} m_{2}^{\prime}$ and $I_{1}^{\prime} \approx_{\ell_{a d v}}^{\bullet} I_{2}^{\prime}$ and $O_{1}^{\prime} \approx_{\ell_{a d v}} O_{2}^{\prime}$

(2) or there is $n^{\prime} \leq n-1$

$$
\left.\triangleleft\left\langle c, m_{2}, I_{2}\right\rangle, O_{\text {init }}, \pi_{\text {init }}, 0 \triangleright \rightarrow{ }_{\tau_{2}}^{n^{\prime}} \backslash\left\langle c_{2}^{\prime}, m_{2}^{\prime}, I_{2}^{\prime}\right\rangle, O_{2}^{\prime}, \pi^{\prime}, n^{\prime}\right\rangle
$$

s.t. $\left(\tau \mid \ell_{a d v}\right)=\left(\tau_{2} \mid \ell_{a d v}\right)$ and $m_{1}^{\prime} \approx_{\ell_{a d v}} m_{2}^{\prime}$ and $I_{1}^{\prime}{\approx_{\ell_{a d v}}^{\bullet}}_{{ }_{2}^{\prime}}^{\prime}$ and $O_{1}^{\prime} \approx_{\ell_{a d v}} O_{2}^{\prime}$ and $\nexists t s^{\prime} \in \operatorname{dom}\left(\pi^{\prime}\right): t s^{\prime} \geq n^{\prime}$ and there is $p c_{1}, p c_{2}$ such that $p c_{1} \nsubseteq \ell_{a d v}$ and $p c_{2} \nsubseteq \ell_{a d v}$ and $c_{1}^{\prime}$ is well-formed w.r.t $\Gamma, p c_{1}, p c^{\prime}$ and and $c_{2}^{\prime}$ is well-formed w.r.t $\Gamma, p c_{2}, p c^{\prime}$

If (1), then we are done by Lemma 5 .

If (2), then by lemma 7 and transitivity we have $m_{1}^{\prime \prime} \approx_{\ell_{a d v}} m_{2}^{\prime}, I_{1}^{\prime \prime} \approx_{\ell_{a d v}}^{\bullet} I_{2}^{\prime}$, and for $\gamma_{1}=\left(t s: \alpha_{1}, \beta_{1}\right)$ that $\left\lfloor\alpha_{1}\right\rfloor_{\ell_{a d v}}^{\bullet}=\epsilon$. Hence by Lemma 9 and transitivity we have $O_{1}^{\prime \prime} \approx_{\ell_{a d v}} O_{2}^{\prime}$ and $\pi^{\prime \prime}=\pi^{\prime}$. We are done by applying Lemma 1 in both runs. 